\title{
Computational Models of Grid Cell Firing
}

\author{
Daniel Bush ${ }^{1,2}$ and Christoph Schmidt-Hieber ${ }^{3}$ \\ ${ }^{1}$ UCL Institute of Cognitive Neuroscience, 17 Queen Square, London, WC1N 3AZ, UK \\ ${ }^{2}$ UCL Institute of Neurology, Queen Square, London, WC1N 3BG, UK \\ ${ }^{3}$ Institut Pasteur, 25 Rue du Docteur Roux, 75724 Paris, France
}

Email correspondence: $\underline{\text { drdanielbush@gmail.com and christoph.schmidt-hieber@pasteur.fr }}$

\section{Overview}

Grid cells in the medial entorhinal cortex $(\mathrm{mEC})$ fire whenever the animal enters a regular triangular array of locations that cover its environment. Since their discovery, several models that can account for these remarkably regular spatial firing patterns have been proposed. These generally fall into one of three classes, generating grid cell firing patterns either by oscillatory interference, through continuous attractor dynamics, or as a result of spatially modulated input from a place cell population. Neural network simulations have been used to explore the implications and predictions made by each class of model, while subsequent experimental data have allowed their architecture to be refined. Here, we describe implementations of two classes of grid cell model - oscillatory interference and continuous attractor dynamics - alongside a hybrid model that incorporates the principal features of each. These models are intended to be both parsimonious and make testable predictions. We discuss the strengths and weaknesses of each model and the predictions they make for future experimental manipulations of the grid cell network in vivo.

\section{Experimental Data}

Grid cells recorded in freely moving rodents fire action potentials at multiple spatial locations. These firing fields form the vertices of a regular triangular array covering the whole environment of a navigating animal (Hafting et al., 2005; Figure 1A). Grid cells were initially discovered in the superficial layers of rodent medial entorhinal cortex (mEC; Hafting et al., 2005; Fyhn et al., 2008), but have since been identified in the pre- and para-subiculum (PreS and PaS; Boccara et al., 2010) and in the deeper layers of $\mathrm{mEC}$, where their firing rate is often modulated by heading direction (Sargolini et al., 2006). Moreover, grid-like responses have been recorded in the parahippocampal cortices of the bat (Yartsev et al. 2011), human (Doeller et al., 2010; Jacobs et al., 2013) and non-human primate (Killian et al., 2012). In the rodent, grid cells are most often recorded in layer II of mEC ( $\mathrm{mECII}$ ), where they are likely comprised of both reelin positive stellate (or 'ocean') cells, which form the majority of principal neurons in $\mathrm{mECII}$ (Gatome et al., 2010), and calbindin positive (or 'island') pyramidal cells (Domnisoru et al., 2013; Kitamura et al., 2014; Ray et al., 2014; Sun et al., 2015).

Grid cell firing patterns can be characterised by their scale (i.e. the distance between adjacent firing fields), orientation (of one principal grid axis relative to an external cue), and the phase or spatial offset of their firing fields (Figure 1A). Grid scale has been shown to increase in discrete steps along 
the dorsoventral axis of mEC (Figure 1B; Barry et al., 2007; Stensola et al., 2012), and evidence suggests that grid cells which share a common scale form a single functional module (Stensola et al., 2012; Yoon et al., 2013). The scale, relative orientation and offset of grid firing patterns within each module are generally conserved across environments (Fyhn et al., 2007), aside from a transient expansion of grid scale in novel environments that returns to baseline with experience (Barry et al., 2012a). The spatial phases of individual grid cells are uniformly distributed across the environment but, importantly, the relative spatial phase of any two simultaneously recorded grid cells from the same module is also conserved across all environments visited by the animal (Fyhn et al., 2007; Yoon et al., 2013).

Principal cells and interneurons in the rodent entorhinal cortex and hippocampus are each modulated by a $5-12 \mathrm{~Hz}$ theta rhythm during movement (Vanderwolf, 1969; O'Keefe and Nadel, 1978). Both the power (Vanderwolf, 1969; McFarland et al., 1975) and frequency (McFarland et al., 1975; Rivas et al., 1996; Jeewajee et al., 2008) of theta oscillations increase with running speed. Importantly, the majority of grid cells in layers II, V and VI of rodent $\mathrm{mEC}$ exhibit theta phase precession, firing spikes at progressively earlier phases of the ongoing movement-related theta rhythm as the grid firing field is traversed (Hafting et al., 2008; Reifenstein et al., 2012; Climer et al., 2013; Jeewajee et al., 2014; Reifenstein et al., 2014; Figure 1C). This theta phase precession appears to be independent of input from the hippocampus (Hafting et al., 2008). Conversely, the majority of layer III grid cells exhibit theta phase locking, firing spikes at the trough of the ongoing theta rhythm throughout the firing field (Hafting et al., 2008; Climer et al., 2013). Interestingly, inactivation of the medial septum, which abolishes the theta rhythm, also impairs grid cell firing patterns while leaving head direction, border and place cell firing patterns unaffected (Brandon et al., 2011; Koenig et al., 2011).

Grid cell firing patterns, like those of place cells, remain stable for a limited period of time in the dark (Hafting et al., 2005). This, along with the fact that grid firing patterns are preserved across all environments visited by the animal, has led to the suggestion that grid cells perform path integration, updating their firing patterns on the basis of self-motion information (Fuhs and Touretzky, 2006; McNaughton et al., 2006). Indeed, grid cell firing patterns are abolished when selfmotion signals are reduced by passive transport of the animal (Winter et al., 2015). However, grid firing patterns of individual cells are also stable between visits to an environment (Hafting et al., 2005; Fyhn et al., 2007), oriented to distal visual cues (Hafting et al., 2005), dependent on visual input (Chen et al., 2016; Perez-Escobar et al., 2016), and rescale parametrically in response to the reshaping of a familiar environment (Barry et al., 2007). This suggests that grid cell firing patterns become anchored to sensory cues with experience, analogous to the reset of a path integration system by sensory inputs to reduce integrated error where possible (Fuhs and Touretzky, 2006; Evans et al., 2016). In addition, theoretical studies have demonstrated that the grid cell population provides a highly efficient code for location (Fiete et al., 2008) and may be used for goal-directed navigation (Bush et al., 2015; Stemmler et al., 2015). 
A
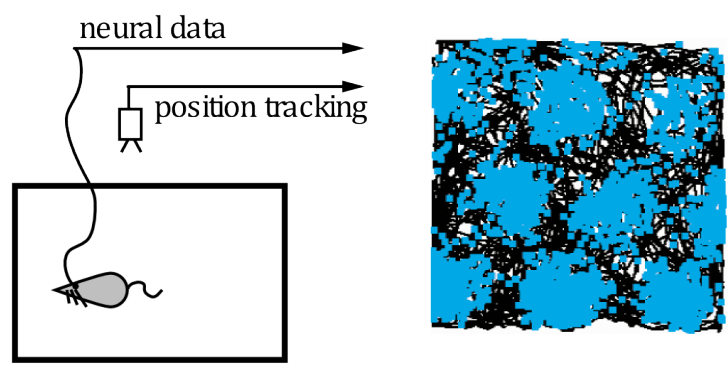

B

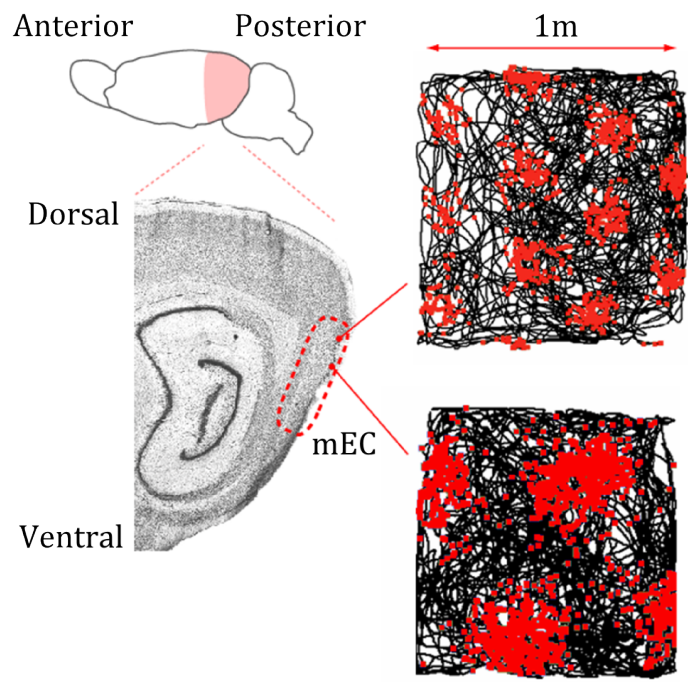

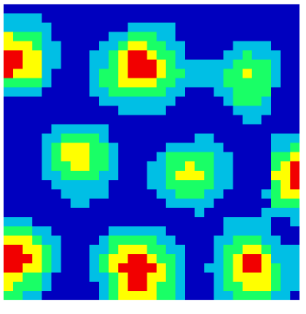

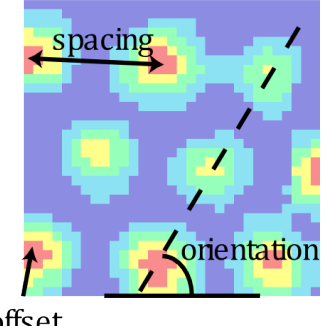

offset

C
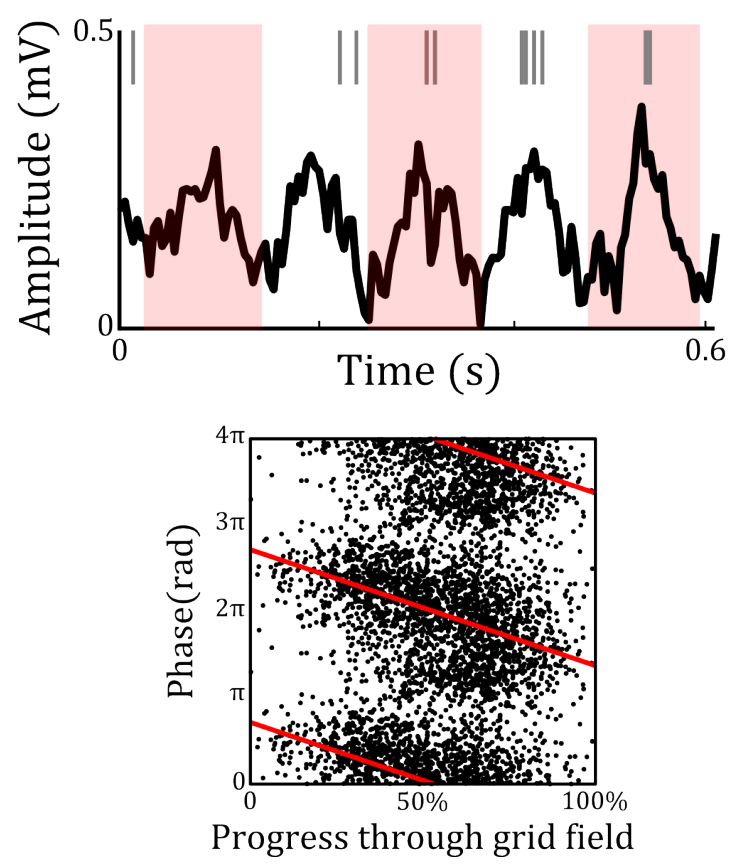

Figure 1: Properties of Grid Cells. (A) The grid cell firing rate code. Left panel: schematic of single unit recording in the freely moving rodent. Middle left panel: the animal's path through the environment is indicated by the black line, and the locations at which action potentials were fired by a single neuron in rodent $\mathrm{MEC}$ are superimposed in blue. Middle right panel: firing rate map for the same $\mathrm{mEC}$ grid cell, with high firing rates indicated by hot colours and low firing rates by cold colours. Right panel: the firing pattern of this $\mathrm{MEC}$ grid cell can be characterised by its scale (the distance between any two adjacent firing fields), orientation (relative to some external cue), and offset or spatial 'phase' (relative to some arbitrary point in the environment; adapted from Bush et al., 2015). (B) Grid cells appear to be organized into discrete functional modules whose scale increases in discrete steps along the dorso-ventral axis of mEC (adapted from Barry et al., 2007). (C) The grid cell temporal code. As the animal crosses a grid firing field, spikes are fired at successively earlier phases of the $5-12 \mathrm{~Hz}$ theta rhythm recorded from the local field potential (LFP), resulting in a negative circular-linear correlation (red line) between the theta phase of firing and progress through the grid field (adapted from Bush et al., 2015).

Recent evidence suggests that sensory inputs to grid cells may be mediated by place and / or boundary cells (Langston et al., 2010; Wills et al., 2010; Bonnevie et al., 2013; Hardcastle et al., 2015; Evans et al., 2016). For example, stable grid cell firing patterns appear after stable head direction (HD), place and boundary cell responses during development, and several days after rats leave the nest and actively explore their environment for the first time (Langston et al., 2010; Wills et al., 
2010). Similarly, inactivating the hippocampus - and thus eliminating place cell inputs to $\mathrm{mEC}$ impairs grid cell firing patterns (Bonnevie et al., 2013). Finally, it has been demonstrated that grid cell firing patterns drift coherently during excursions into the centre of an open field environment, and that this accumulated error is eliminated by contact with environmental boundaries (Hardcastle et al., 2015). This is complemented by the observation that environmental boundaries have a strong influence on the orientation and ellipticity of grid cell firing patterns (Derdikman et al., 2009; Krupic et al., 2015) that develops with experience (Stensola et al., 2015). Each of these effects is likely to be mediated by input from boundary cells (Barry et al., 2006; Savelli et al., 2008; Solstad et al., 2008; Lever et al., 2009).

\section{The Models}

Any computational model of grid cell firing patterns must account for the experimental data described above whilst remaining faithful to the known neurobiology of the medial entorhinal cortex. Ideally, such a model should replicate both the rate and temporal code exhibited by grid cells - that is, generate both a triangular lattice of spatial receptive fields and phase precession against an ongoing oscillation in the local field potential. In accordance with their hypothesised role in path integration, most models assume that the principal input to grid cells is a self-motion signal corresponding to the animal's velocity. However, to account for the stability of their firing patterns relative to the environment, grid cells must also receive environmental sensory inputs from place or boundary cells which may have an effect on those firing patterns. Finally, the stability of grid firing patterns relative to one another, and their modular organisation, also suggest that there are strong, local interactions between grid cells in $\mathrm{mEC}$.

A number of grid cell models that can account for some or all of these properties have been proposed, each differing in how the animal's location is encoded, updated, and decoded (reviewed by Giocomo et al., 2011; Zilli, 2012). These models are not mutually exclusive, however, and the properties of grid cells may best be accounted for by a 'hybrid' model that incorporates features from each class (Schmidt-Hieber and Häusser, 2013; Bush and Burgess, 2014). Grid cell models can be broadly divided into several principal classes. The first class are oscillatory interference (OI) models, which hypothesise that grid cell firing patterns are formed at the single cell level by constructive interference (i.e. coincidence detection) among velocity controlled oscillator (VCO) inputs (Burgess et al., 2005, 2007; Blair et al., 2008; Burgess, 2008; Hasselmo, 2008). The second class are continuous attractor network (CAN) models, which hypothesise that grid cell firing patterns are generated at the network level by local interactions characterised by a circular surround synaptic weight profile (Fuhs and Touretzky, 2006; McNaughton et al., 2006; Guanella et al., 2007; Burak and Fiete, 2009; Pastoll et al., 2013). Another class of models suggest that grid cell firing patterns arise at the single cell level as a result of spatially modulated inputs from place cells, performing a process equivalent to principal component analysis (PCA) on the spatial representation provided by the hippocampus (Kropff and Treves, 2008; Dordek et al., 2016). Finally, grid cell firing may emerge as a result of a self-organizing learning process (Mhatre et al., 2012).

Here, we describe neural network models of two of these major classes of grid cell model, using either oscillatory interference or continuous attractor dynamics to generate grid firing patterns, alongside an appraisal of their strengths, weaknesses and relationship to experimental data. We 
then present a hybrid model that incorporates features of each class in order to account for a wider array of the known properties of grid cells. Finally, we provide suggestions for future experimental studies that will help to further refine the biological validity of grid cell models, and critical tests of each model.

\section{The Oscillatory Interference Model}

The oscillatory interference $(\mathrm{OI})$ model was originally proposed to account for theta phase precession in place cells (O'Keefe and Recce, 1993; Lengyel et al., 2003). This model proposes that grid firing patterns can be accounted for at the single cell level by constructive interference between two or more oscillatory inputs (Burgess et al., 2005; Burgess et al., 2007; Blair et al., 2008; Burgess, 2008; Hasselmo, 2008). In its simplest 1D form, one oscillator is assumed to have a constant baseline frequency, and the other "velocity controlled oscillator" (VCO) is assumed to have a frequency that varies linearly with the speed of movement (Equation 1; Burgess, 2008). In rodents, the baseline frequency is generally assumed to be the $5-12 \mathrm{~Hz}$ movement related theta oscillation (Vanderwolf, 1969; O'Keefe and Nadel, 1978; Burgess et al., 2007).

$$
\begin{gathered}
\mathbf{v}_{\mathrm{VCO}}(t)=s(t) \cos \left(\varnothing(t)-\emptyset_{V C O}\right) \\
f_{V C O}(t)=f_{\text {base }}+\beta \mathbf{v}_{\mathrm{VCO}}(t)
\end{gathered}
$$

Equation 1: The relationship between VCO burst firing frequency and movement velocity. VCO burst firing frequency $f_{v c o}$ deviates linearly from the baseline frequency $f_{\text {base }}$ according to the component of movement velocity along the VCO's preferred direction $\mathbf{v}_{\mathrm{vcO}}$, which is dictated by the absolute speed $s$ and direction $\emptyset$ of the animal's movement relative to the preferred direction of that $V C O \emptyset_{V C O}$. The scale of the resultant grid firing pattern is dictated by the slope of the linear relationship between burst firing frequency and velocity, $b$.

Input from these two signals generates grid cell membrane potential oscillations (MPOs) which are modulated by an "envelope" frequency that is equal to the difference in baseline and VCO frequencies; and a "carrier" frequency that is equal to the mean of those two frequencies (Figure $2 \mathrm{~A})$. The envelope corresponds to the grid cell rate code - being spatially periodic and approximately Gaussian or cosine tuned; while the carrier corresponds to the temporal code - being higher in frequency than the baseline oscillation, and thus causing the grid cell to fire at progressively earlier phases of that baseline oscillation as the firing field is traversed (i.e. generating phase precession; Figure 2A). The scale of the resultant grid firing pattern is controlled by the slope of the VCO movement speed / burst firing frequency relationship $\beta$, which determines how quickly the VCO and baseline oscillation move in and out of phase during movement. 


\section{A}
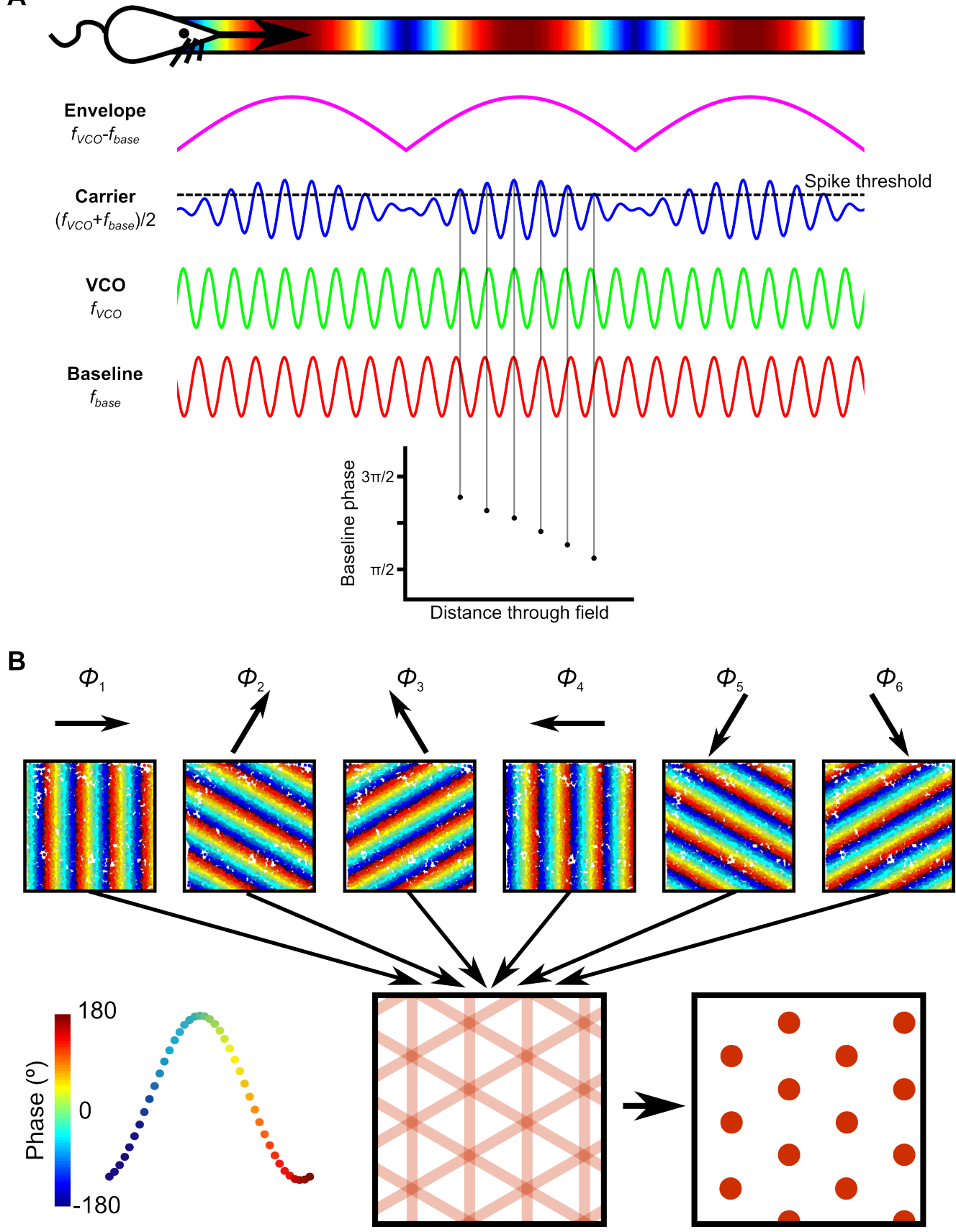

Figure 2: The Oscillatory Interference Model. (A) In 1D, consider a baseline oscillation with frequency $f_{\text {base }}$ (red line) and a velocity controlled oscillation (VCO) with a frequency $f_{V c o}$ (green line) that varies linearly with movement speed. Constructive interference between these two oscillations generates a spatially periodic activity pattern with a carrier frequency (blue) equal to their mean frequency $\left(f_{\text {base }}+f_{v c o}\right) / 2$ and an envelope frequency (pink) equal to their difference in frequency $\left(f_{v c o}-f_{\text {base }}\right)$. This activity pattern corresponds to spatially periodic, approximately Gaussian firing fields (top) within which spikes are fired at progressively earlier phases of the baseline oscillation (bottom), as observed in grid cells (following Blair et al., 2008). (B) Velocity controlled oscillators (VCOs) with different preferred firing directions $\emptyset_{i}$ encode periodic displacement along that direction in their firing phase. Combining input from two or more VCOs with preferred firing directions that differ by multiples of $60^{\circ}$ can then account for the periodic firing fields exhibited by grid cells, which fire when their VCO inputs are in phase (adapted from Bush and Burgess, 2014). 
The OI model can be extended to account for grid firing patterns in 2D by incorporating input from multiple VCOs whose burst firing frequencies vary linearly with movement speed along different preferred directions. Because distance is the time integral of velocity, and phase is the time integral of frequency, the phase of each VCO - if sampled at fixed intervals (i.e. at the peak or trough of the baseline oscillation) - encodes (periodic) displacement in its preferred direction (Figure 2B). A grid cell that receives input from two or more VCOs whose preferred directions differ by multiples of $60^{\circ}$ will exhibit a triangular array of firing fields at locations where those VCO inputs are in phase (Figure 2B). The specific location or offset of those firing fields can be manipulated by adding a constant phase shift to one or more VCO inputs. Hence, the OI model proposes that each VCO performs path integration along different one-dimensional axes, while grid cells simply "read-out" the activity of multiple VCO inputs by firing whenever they are in phase (Figure 2B). Importantly, it is the phase difference between VCO and baseline oscillations that encodes location, and the baseline oscillation can therefore take any frequency value and need not be constant over time (Burgess, 2008; Blair et al., 2014; Orchard, 2015).

What is the source of these VCO inputs to grid cells? Early implementations of OI grid cell models suggested that spontaneous independent intrinsic oscillations in dendritic subunits, driven by animal velocity, may represent VCOs ("intrinsic VCOs"; Burgess et al., 2007). This idea was inspired by the finding that $\mathrm{mECll}$ stellate cells can produce spontaneous somatic membrane potential oscillations in the theta frequency range (theta MPOs) when depolarised close to spike threshold (Alonso and Llinas, 1989). Moreover, the frequency of these MPOs, along with several intrinsic membrane properties, shows a dorsal-ventral gradient (Giocomo et al., 2007; Garden et al., 2008) that mirrors the parallel anatomical gradient in grid spacing (Hafting et al., 2005). However, as biophysical modelling has revealed that dendritic intrinsic MPOs will rapidly phase lock (Remme et al., 2010), more recent $\mathrm{OI}$ model implementations assume that VCOs are represented by neurons projecting to grid cells that display velocity-dependent theta-modulated firing ("external VCOs"; Burgess, 2008; Welday et al., 2011; Schmidt-Hieber and Häusser, 2013; Bush and Burgess, 2014).

How can we predict membrane potential $V_{m}$ and spike rate from an Ol model of grid cell firing? The simplest way is to analytically compute $V_{m}$ for an Ol model neuron that receives input from $n$ VCOs with preferred firing directions that differ by multiples of $60^{\circ}$, each oscillating at frequency $f_{V C O, i}$ (see Equation 1) according to Equation 2.

$$
V_{m}(t)=\prod_{i=1}^{n}\left[\cos \left(2 \pi f_{\text {base }} t\right)+\cos \left(2 \pi f_{V C O, i}(t) t\right)+\varphi_{i}\right]_{+}
$$

Equation 2: Membrane potential of a grid cell simulated using the Ol model. The membrane potential $V_{\mathrm{m}}$ of the simulated grid cell at time step $t$ is dictated by the baseline frequency $f_{\text {base }}$, VCO burst firing frequency $f_{V C O, i}$ (see Equation 1) and spatial phase offset of the $i^{\text {th }} \vee \mathrm{VCO} \varphi_{i}$, with $[x]_{+}=\max \{0, x\}$ (Burgess et al., 2007; Burgess, 2008)

Figure $3 \mathrm{~A}$ shows an example of the membrane potential $V_{m}$ of a simulated grid cell in a $2 \mathrm{D}$ environment generated by two VCO inputs with preferred directions $\emptyset_{V C O, 1}=0^{\circ}$ and $\emptyset_{V C O, 2}=60^{\circ}$. Although two VCO inputs and a baseline oscillation are sufficient to produce a hexagonal grid, more 
circular firing fields that better approximate experimental recordings can be generated by six VCO inputs with preferred firing directions that differ by multiples of $60^{\circ}$ (Burgess et al., 2007). This configuration is also necessary to produce omnidirectional phase precession in $2 \mathrm{D}$, if the firing rate of VCOs is directionally tuned such that VCOs only fire spikes when movement velocity in their preferred direction is positive - i.e. when the running direction does not exceed $\emptyset_{V C O, i} \pm 90^{\circ}$ (Burgess, 2008; Climer et al., 2013).

To obtain a more biophysically realistic estimate of grid cell activity, integrate-and-fire neurons (Welday et al., 2011; Bush and Burgess, 2014) or detailed compartmental modelling (Schmidt-Hieber and Häusser, 2013) have been employed. These implementations typically convert $f_{V C O, i}$, which is continuous in time, into discrete spike trains driving synaptic inputs to a model neuron. For example, discrete Poisson firing trains can be generated by computing the firing probability $p_{V C O, i}$ for $\mathrm{VCO}_{i}$ according to Equation 3, where the time step $\Delta t$ is chosen so that $p \ll 1$. Predictions for the membrane voltage $V_{m}$ and spike rate from a detailed compartmental model are shown in Figure 3B (adapted from Schmidt-Hieber and Häusser, 2013). While an isolated single-cell Ol model fails to reproduce the experimentally recorded membrane potential ramps during firing field crossings (Figure $3 \mathrm{C}$ ), it can account for the observed phase precession phenotype, with both action potentials and theta membrane potential oscillations showing phase precession with reference to LFP theta (Figure 3D, E).

$$
p_{V C O, i}(t, t+\Delta t)=\overline{r_{V C O}}\left[\cos \left(2 \pi f_{V C O, i}(t) t+\varphi_{V C O, i}\right)+1\right] \Delta t
$$

Equation 3: Simulated VCO spike train in the OI model of grid cell firing. The probability of an input spike in time step $t$ is dictated by VCO mean firing frequency $\overline{r_{V C O}}$, burst firing frequency $f_{V C O, i}$ (see Equation 1 ), the spatial phase of that VCO input $\varphi_{V C O, i}$ and length of the time step $\Delta t$. Firing probability is converted to input spikes by drawing a random number $r$ from the interval $[0,1]$ for each time step. A spike is produced if $r \leq p$. 
A

Simple model
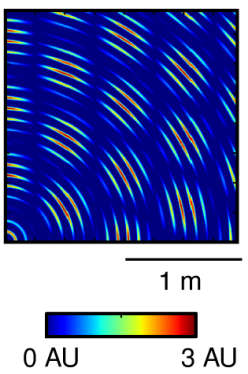

C

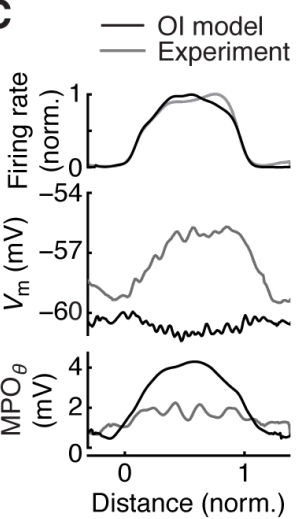

B

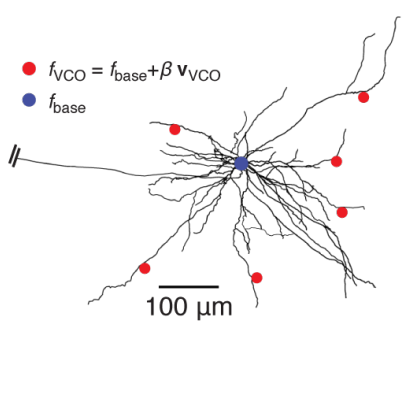

D

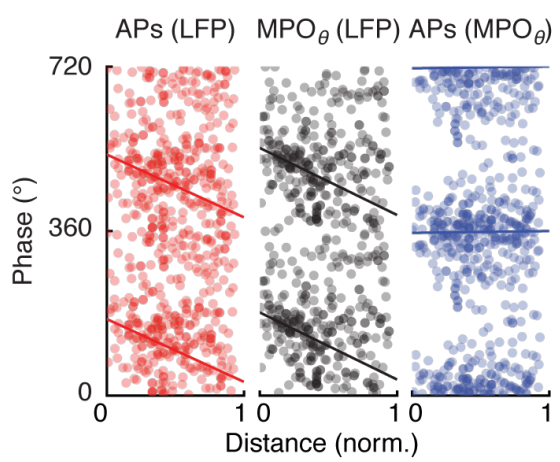

Compartmental model

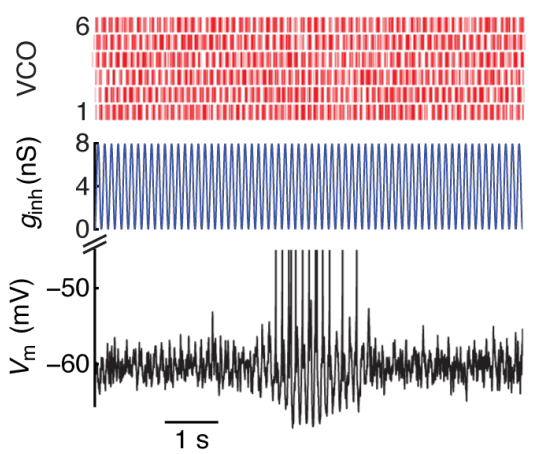

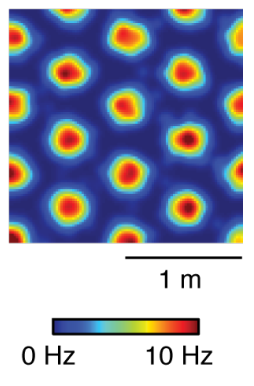

E

Model

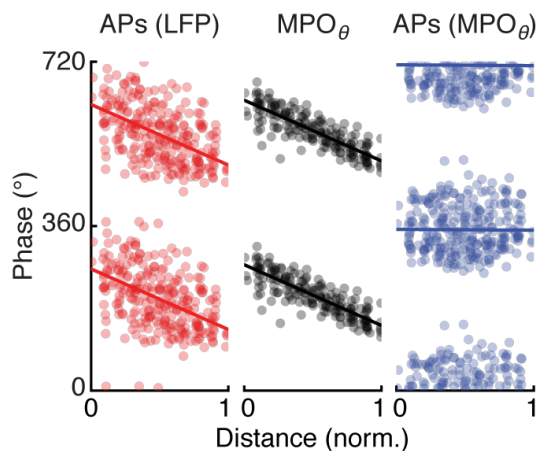

Figure 3: Implementations of the Oscillatory Interference Model. (A) In the simple implementation, following Equation 2, the membrane voltage $V_{m}$ of a simulated grid cell is generated by $2 \mathrm{VCO}$ inputs with preferred directions of $\emptyset_{V C O, 1}=0^{\circ}$ and $\emptyset_{V C O, 2}=60^{\circ}$. (B) In a more detailed implementation, Poissonian VCO spike trains, following Equation 3, are used as input to six excitatory synapses (red circles) located on the distal dendrites of a compartmental stellate cell model, while the baseline oscillation takes the form of an inhibitory conductance $g_{\text {inh }}$ applied directly to the soma (blue circle). In both cases, the results shown here were generated by 90 linear runs with $s=0.2 \mathrm{~ms}^{-1}$ from the bottom left corner to the opposing boundaries of a $2 \mathrm{~m}$ sided square arena at angles spaced by $1^{\circ}$. For further details, see Schmidt-Hieber and Häusser (2013); code is available on ModelDB (accession number 150239). (C) Simulations demonstrate a discrepancy between the predictions of the OI model and whole cell recordings of grid cell membrane potential in behaving animals (Schmidt-Hieber and Häusser, 2013; Domnisoru et al., 2013). The OI model predicts no change in the average membrane voltage of a grid cell (middle panel) as the firing field is traversed (top panel), but does predict an increase in the amplitude of theta band membrane potential oscillations $\left(M P O_{\vartheta}\right)$ as VCO inputs become synchronised in the centre of the firing field (bottom panel). Conversely, experimental recordings indicate a sustained 'ramp' depolarisation that mirrors the change in firing rate within the grid field, but no change in the amplitude of theta band MPOs. (D) Simulations demonstrate that the predictions of the Ol model are consistent with the properties of phase precession described by whole cell recordings of grid cell membrane potential in behaving animals (Schmidt-Hieber and Häusser, 2013; Domnisoru et al., 2013). The phases of action potentials (APs) with respect to LFP theta (left), theta band MPOs with respect to LFP theta (middle) and action potentials with respect to theta band MPOs (right) are plotted as a function of normalized position within firing fields of stellate cells recorded intracellularly in vivo. Action potentials show phase precession with respect to the LFP, but are phase locked with MPOs. (E) The compartmental model correctly predicts the experimental observations. Panels D-E adapted from Schmidt-Hieber and Häusser (2013). 


\section{Critique of the Oscillatory Interference Model}

The Ol model accounts for both the rate and temporal firing patterns of grid cells - that is, it generates both hexagonally arranged firing fields and phase precession. In accordance with the OI model, grid cell burst firing frequency has been shown to increase with running speed (Jeewajee et al., 2008) and decrease in novel environments, when grid scale expands (Barry et al., 2012a; Wells et al., 2013). Moreover, cells with VCO-like properties have been identified in and around the entorhinal cortex (Welday et al., 2011); gridness scores correlate with spike train theta rhythmicity (Boccara et al., 2010); and grid cell firing patterns are impaired when theta power is reduced by inactivation of the medial septum (Brandon et al., 2011; Koenig et al., 2011) or when the influence of running speed on theta frequency is abolished by passive transport of the animal (Winter et al., 2015).

The OI model has recently been challenged by the finding that grid cell firing patterns in crawling bats exist in the absence of a continuous theta rhythm (Yartsev et al., 2011, but see Barry et al., 2012b). Similarly, continuous low frequency oscillations are rarely observed in the human hippocampal formation during virtual navigation tasks (Ekstrom et al., 2005; Jacobs et al., 2013; Watrous et al., 2013). However, it is important to note that the OI model functions equally well with a baseline oscillation of any frequency, which need not be constant over time, as integrated displacement is encoded in the phase difference between baseline and VCO oscillations irrespective of the absolute phase or frequency of either oscillation (Burgess, 2008; Blair et al., 2014; Orchard, 2015). Indeed, several computational models of grid cells effectively make use of oscillatory interference with a baseline frequency of $\mathrm{OHz}$, in which case each $\mathrm{VCO}$ is equivalent to a nonoscillating 'stripe' or 'band' cell (Mhatre et al., 2012; Horiuchi and Moss, 2015). Moreover, recent recordings from bats have revealed that, despite a lack of clear LFP rhythmicity, neurons still exhibit phase precession with respect to broadband low-frequency oscillations in the non-rhythmic LFP (Eliav et al., 2015). These data can be accounted for by an OI model with a baseline oscillation that varies dynamically over a wide range. Hence, the absence of any clear, sustained oscillatory activity in the LFP or grid cell spike train is not sufficient to disprove the Ol model.

The OI model has also been criticised as being particularly susceptible to noise in the burst firing frequency of VCO inputs (Welinder et al., 2008). However, the phase precession of grid and place cell firing demonstrates that oscillations with the precise timing required to generate grid cell firing patterns by oscillatory interference do exist in the rodent hippocampal formation (O'Keefe and Recce, 1993; Hafting et al., 2008). It is well known that grid firing patterns - like any hypothetical path integration system - will rapidly accrue error over time in the absence of sensory inputs (Hafting et al. 2005; Evans et al., 2016). Theoretical studies have demonstrated that input from place or boundary cells is sufficient to stabilise grid firing patterns in the face of phase noise (Bush and Burgess, 2014). Similarly, the OI model has been criticised for relying on the preferred direction of VCO inputs to a grid cell being separated by multiples of $60^{\circ}$, but theoretical studies have shown that such inputs may be selected by a Hebbian learning mechanism during development as they most frequently co-occur in space (Burgess et al., 2007; Mhatre et al., 2012) and therefore offer optimal noise reduction (Burgess and Burgess, 2014).

The OI model cannot, however, account for the relative stability of grid cell firing patterns within a module (Yoon et al., 2013). Oscillatory interference is a single cell model, and makes no comment on 
potential interactions between grid cells, while experimental evidence demonstrates that grid cell firing patterns from the same functional module are tightly coupled, responding coherently to changes in a familiar environment and maintaining their relative spatial phase between environments (Hafting et al., 2005; Barry et al., 2007; Fyhn et al., 2007; Stensola et al., 2012; Yoon et al., 2013). Organising VCO inputs into ring attractor circuits provides some stability between firing patterns of different grid cells (Blair et al., 2008), but overlooks the functional consequences of the known synaptic interactions between grid cells. Similarly, the observations of an in-field 'ramp' depolarisation in grid cell subthreshold membrane potentials, as well as the lack of increase in infield theta amplitude, are not consistent with an Ol model (Figure 3C; Domnisoru et al., 2013; Schmidt-Hieber and Häusser, 2013). Hence, some modification of the model or additional mechanism is required to account for both interactions between grid cells within each functional module, and the subthreshold membrane potential dynamics of grid cells recorded in vivo.

\section{Continuous Attractor Network Models}

Continuous attractor network (CAN) models were originally proposed to account for the properties of head direction (Zhang, 1996) and place cells (Samsonovich and McNaughton, 1997; Conklin and Eliasmith, 2005). This class of model proposes that spatially modulated firing patterns can be accounted for by local, recurrent interactions among cells (Fuhs and Touretzky, 2006; McNaughton et al., 2006; Guanella et al., 2007; Burak and Fiete, 2009). The requisite recurrent connectivity is characterised by a circular, centre surround synaptic weight profile on a topographically arranged sheet of neurons (Figure 4A). In the case of grid cells, this implies that the strength of synaptic connections between cells decreases as a function of the distance between their firing fields (i.e. the difference in spatial phase). This establishes cooperation between grid cells with similar spatial phase, and competition between grid cells with different spatial phases (Fuhs and Touretzky, 2006). Note that grid cells need not be topographically arranged in the actual brain, this formalism is introduced purely to aid visualisation (but see Naumann et al., 2015).

As direct recurrent excitatory connections between grid cell candidate neurons in $\mathrm{mECll}$ are sparse, recurrent connectivity in most CAN model implementations is mediated by disynaptic inhibition from interneurons, which have been shown to densely innervate $\mathrm{mECll}$ principal neurons (Dhillon and Jones, 2000; Couey et al., 2013; Pastoll et al., 2013; Fuchs et al., 2016). Uniform excitatory input to such a network will generate one or more stable activity packets or 'bumps', and self-motion information can then be used to translate the position of this activity packet across the neural sheet in accordance with the animal's movement in the real world. The majority of CAN models suggest that the activity bump is shifted by asymmetric interactions between grid cells in the neural sheet. This can be achieved by rate-coded input from conjunctive grid $\mathrm{x}$ movement direction cells, which have also been identified in the deeper layers of $\mathrm{mEC}$ (Sargolini et al., 2006). If the recurrent inhibitory input from these conjunctive cells to other cells in the network is shifted along the axis of their preferred firing direction, then their firing will shift the activity bump in the movement direction (Figure 4B). 

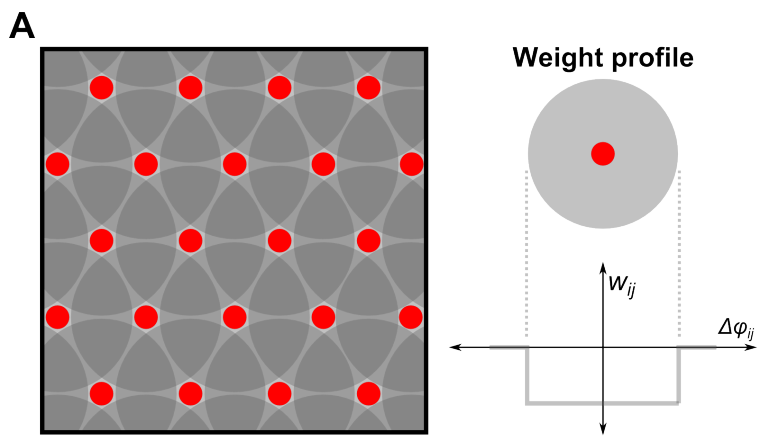

B
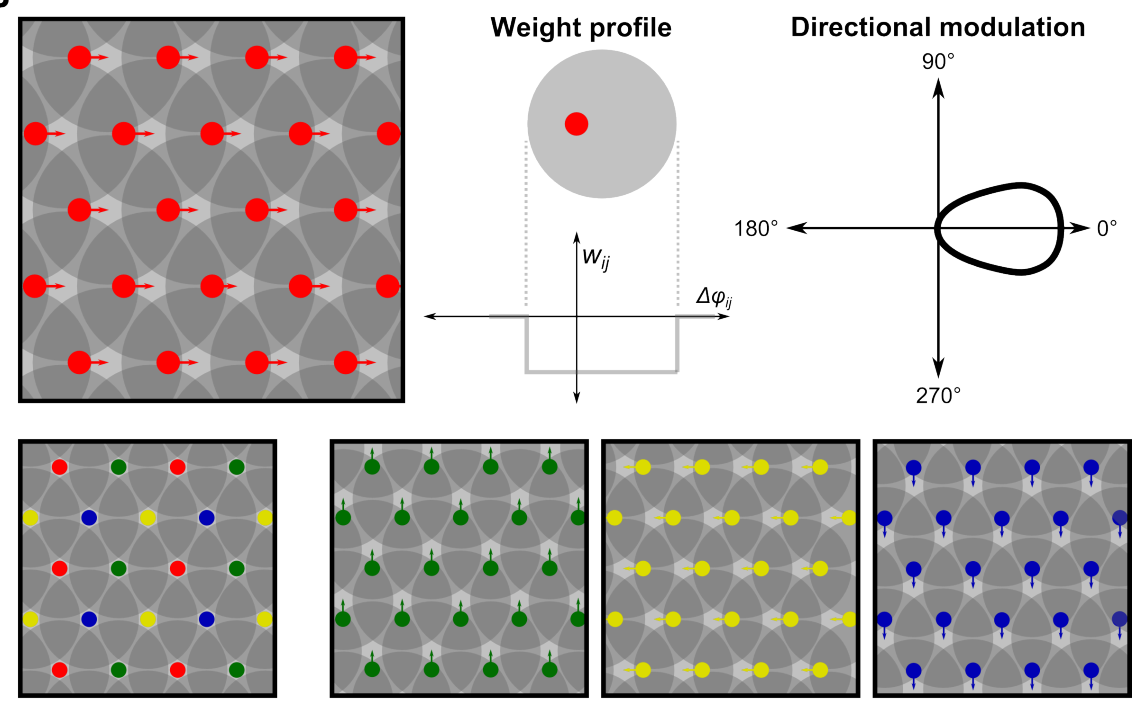

C
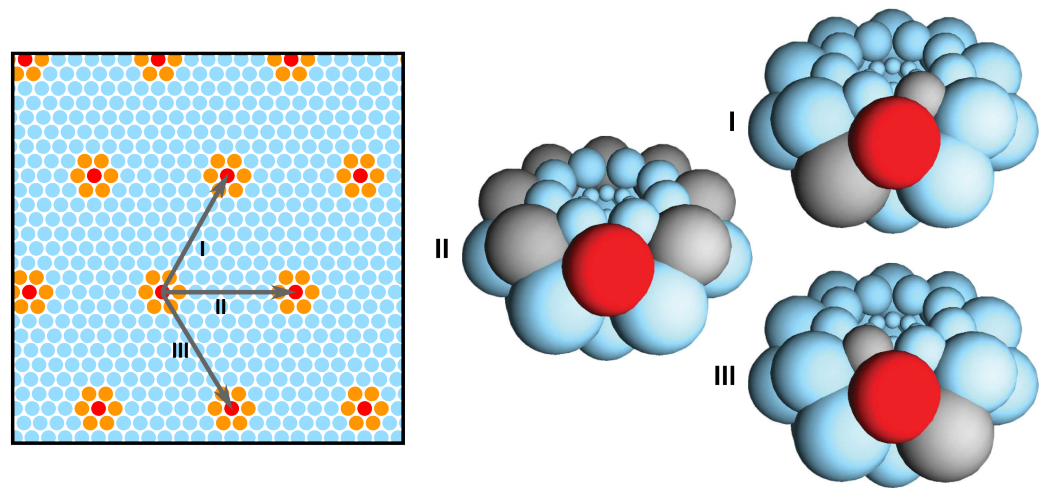

Figure 4: The Continuous Attractor Network Model. (A) A sheet of topographically arranged neurons (left panel) are connected by disynaptic inhibitory projections with a circular surround profile (right panel), such that neurons which are proximate on the neural sheet inhibit each other. In the multiple bump CAN model, uniform excitatory input to such a network generates a grid firing pattern, as activity bump(s) form at the triangular array of inhibitory minima produced by close packing of the circular connectivity profile. In the single-bump CAN model, not illustrated here, a single activity bump forms at an arbitrary location on the neural sheet. Note that neurons need not be topographically arranged in the actual brain. (B) The location of the(se) activity bump(s) can be shifted by asymmetric interactions between grid cells. For example, if conjunctive grid cells, whose firing rate is modulated by movement direction, have recurrent inhibitory connections that are skewed along their preferred firing direction, then their activity will create inhibitory minima just ahead of the activity bump(s) in that direction on the neural sheet. The activity bump(s) will subsequently move across the neural sheet, tracking the animal's movement in the real world. A mixture of conjunctive grid cells with different preferred firing directions can therefore both establish and shift the grid firing pattern in any direction (following Burak and Fiete, 2009). (C) To account for smooth changes in grid cell firing over large distances, a periodic continuous attractor network must adopt a twisted torus topology, such 
that movement of the activity bump along orientations that differ by multiples of $60^{\circ}$ will return the activity bump to its original location on the sheet of cells once some integer number of grid scales have been travelled. Hence, if a grid cell is active at some location in the real world (red circle, left panel), then it will also be active at a fixed distance equal to the grid scale along any grid axis. Similarly, if an activity bump is located over some grid cell on a neural sheet that exhibits a twisted torus topology (red sphere, right panel), then movement of a fixed distance equal to the grid scale along any grid axis will return the activity bump to its original location (adapted from Bush et al., 2015).

In the case of a single activity bump, the network must exhibit a twisted torus topology, such that movement of a set distance in a direction corresponding to any multiple of $60^{\circ}$ across the neural sheet will return it to its original position, thus accounting for the hexagonal symmetry of the grid firing pattern in the real world (Figure 4C; Guanella et al., 2007; Pastoll et al., 2013). In the case of multiple bumps, the circular weight profile dictates that the location of activity bumps on the neural sheet exhibit six-fold symmetry through circular close packing. To ensure that activity bumps smoothly appear and disappear at the edges of the neural sheet, either periodic boundary conditions are imposed (which places constraints on the dimensions of the neural sheet), or alternatively the synaptic weights (Fuhs and Touretzky, 2006) or feedforward synaptic inputs (Burak and Fiete, 2009) are smoothly modulated to zero towards the edges of the neural sheet. Importantly, population activity is constrained by the synaptic connections between neurons such that grid cell firing patterns can only ever encode a single location at any time. Hence, grid cells in the continuous attractor network effectively perform path integration, tracking the animal's location by integrating self-motion signals.

An influential rate-based implementation of a multiple bump CAN model was proposed by Burak and Fiete (2009). In this model, each neuron $i$ is arranged on a rectangular sheet and assigned one of four preferred directions $\left(\emptyset_{i}=0^{\circ}, 90^{\circ}, 180^{\circ}\right.$, or $\left.270^{\circ}\right)$. Typical sizes of the neuronal sheet range from $40 \times 40$ to $256 \times 256$ neurons - larger networks provide higher integration accuracy (Burak and Fiete, 2009). The neuronal dynamics of each simulated grid cell are described by Equation 4 , where $r_{i}$ is the firing rate of neuron $i, \tau$ is the integration time constant, $R_{i}$ are recurrent inputs and $B_{i}$ are feedforward inputs. Recurrent inputs $R_{i}$ are equal to the sum of all presynaptic firing rates $r_{j}$ multiplied by the corresponding recurrent synaptic weights $w_{i j}$.

$$
\begin{gathered}
\tau \frac{d r_{i}}{d t}+r_{i}=\left[R_{i}+B_{i}\right]_{+} \\
R_{i}=\sum_{j} w_{i j} r_{j}
\end{gathered}
$$

Equation 4: Firing rate dynamics of a grid cell simulated using the CAN model. The firing rate $r_{i}$ of neuron $i$ is dictated by the time constant $\tau$, recurrent input $R_{i}$ and feedforward inputs $B_{i}$, with $[x]_{+}=\max \{0, x\}$. Recurrent inputs are equal to the sum of all pre-synaptic firing rates $r_{j}$ multiplied by the corresponding recurrent synaptic weights $w_{i j}$ (Burak and Fiete, 2009).

The strength of recurrent inputs $w_{i}$ depends on the distance between the 2D positions $\mathbf{x}_{i}$ and $\mathbf{x}_{j}$ of pre- and post- synaptic neurons $j$ and $i$ on the neuronal sheet; and is shifted by a vector $l \hat{\mathbf{u}}_{\phi_{j}}$, where 
$\hat{\mathbf{u}}_{\phi_{j}}$ is a unit vector in the preferred direction $\emptyset_{j}$ and $/$ defines the amplitude of the shift (typically, a small number e.g. 2 neurons; Equation 5). The dependence of this shift on $\emptyset_{j}$, the preferred direction of the pre-synaptic neuron, indicates that it is applied to the outgoing weights. The centresurround synaptic weight matrix $\mathbf{W}_{0}$ classically takes the shape of a "Mexican hat", with excitation dominating the centre and inhibition forming a brim in the periphery (Fuhs and Touretzky, 2006). This can be generated as a difference of Gaussians (Equation 5B), where $a$ defines the amplitude and $\gamma$ the width of the excitatory centre, and $\kappa$ determines the width of the inhibitory brim.

In the original implementation, the recurrent weight matrix was purely inhibitory (using $a=1$ ). Interestingly, this implementation was suggested before detailed analysis of the functional connectivity in $\mathrm{mECll}$ revealed that direct excitatory recurrent connections between principal cells are sparse or lacking (Couey et al., 2013; Pastoll et al., 2013; Fuchs et al., 2016). Following more detailed analysis of the connectivity between $\mathrm{mECll}$ stellate cells, variations of the centre-surround connectivity matrix with steep edges that resemble a frying pan or 'Lincoln Hat' have also been used in CAN models (as illustrated in Figure 4; Couey et al., 2013).

$$
\begin{gathered}
w_{i j}=f\left(\mathbf{x}_{i}-\mathbf{x}_{j}-l \hat{\mathbf{u}}_{\phi_{j}}\right) \\
f(\mathbf{x})=a e^{-\gamma|\mathbf{x}|^{2}}-e^{-\kappa|\mathbf{x}|^{2}}
\end{gathered}
$$

Equation 5: Recurrent synaptic weight profile in the CAN model of grid cell firing. The strength of recurrent connectivity $w_{i j}$ between pre-synaptic neuron $j$ and post-synaptic neuron $i$ is a function of the distance between their 2D locations $\mathbf{x}_{i}$ and $\mathbf{x}_{j}$ on the neural sheet, and is skewed along the preferred direction $\emptyset_{j}$ of the pre-synaptic neuron according to the product of a constant $/$ and unit vector in that direction $\hat{\mathbf{u}}_{\phi_{j}}$. In canonical implementations of the CAN model, synaptic weights follow a 'Mexican hat' profile, consisting of excitatory projections to proximate neurons and inhibitory projections to more distant neurons on the neuronal sheet. This can be generated by a difference of Gaussian distributions, where $a$ defines the amplitude of synaptic weights, $v$ and $k$ control the width of the excitatory and inhibitory components, respectively.

Finally, feedforward inputs in the CAN model $B_{i}$ are modulated by the animal's running direction, as defined by Equation 6, where $v$ is the animal's velocity and $\alpha$ determines the amplitude of the directional modulation of feedforward input. Hence, coupling of network activity to the animal's trajectory is realised through the combination of two mechanisms: a neuron receives more feedforward input if the animal is running in its preferred direction (Equation 6); and the outgoing weights of each neuron are shifted by a small number of neurons along that preferred direction (Equation 5). As a consequence, if the animal is running in a certain direction, neurons that prefer this direction (or components of this direction) will be activated by their feedforward input and can then impose a shift in network activity towards their preferred direction. In contrast, neurons with preferred directions that point away from the running direction will receive less feedforward input, and their outgoing weights, which are shifted in the opposite direction, will be less effective. Importantly, attractor dynamics ensure that the firing rate of these cells may not be significantly modulated by running direction (Bonnevie et al., 2013), such that they would not be classed as conjunctive cells if recorded experimentally (Sargolini et al., 2006). 


$$
B_{i}=1+\alpha \hat{\mathbf{u}}_{\phi_{i}} \cdot \mathbf{v}
$$

Equation 6: Directionally modulated external input in the CAN model of grid cell firing. In order to shift the activity bump in concert with the animal's movement in the real world, external input to a simulated grid cell is modulated by the velocity of movement $v$ multiplied by a unit vector $\hat{\mathbf{u}}$ in the preferred direction $\emptyset_{j}$ of that grid cell and a positive constant $\alpha$. The outgoing synaptic weight profile of each grid cell is also skewed along the preferred firing direction (Equation 5), creating an inhibitory minimum adjacent to the activity bump in that direction on the neural sheet.

A

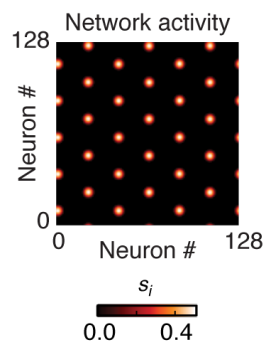

Simple model

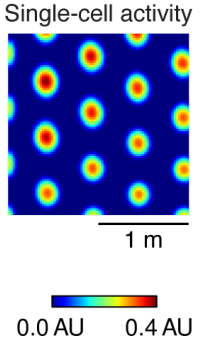

B

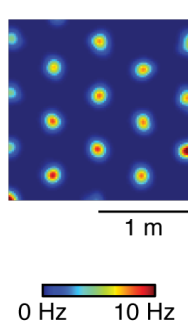

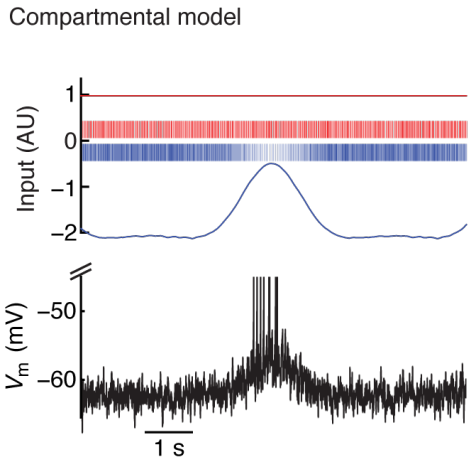

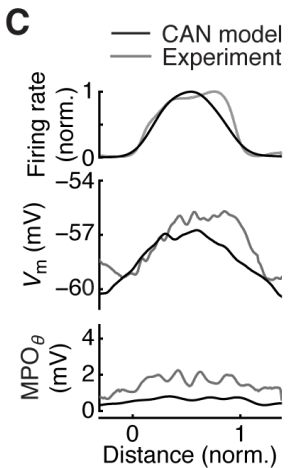

Figure 5: Implementations of the Continuous Attractor Network Model. (A) In the simple implementation, $128^{2}$ rate-based neurons are arranged on a neural sheet with periodic boundary conditions and Mexican-hat connectivity as described by Equation 5 . The left panel shows activity of each neuron in network space at a given moment in time. Note the periodic activity bumps that form spontaneously in the network. The right panel shows a colour-coded spatial map of the activity of an individual neuron averaged across time during simulated navigation within a square-shaped environment. (B) In a more detailed implementation, the probabilities of excitatory (red) and inhibitory (blue) input spikes in each time step are derived from the simple rate-based model in $(\mathrm{A})$ and used to generate input to a compartmental stellate cell model. For further details see Schmidt-Hieber and Häusser (2013); code is available on ModelDB (accession number 150239). (C) Simulations demonstrate that the predictions of the CAN model closely match whole cell recordings of grid cell membrane potential in behaving animals (Schmidt-Hieber and Häusser, 2013; Domnisoru et al., 2013). The CAN model predicts a ramped depolarisation of the grid cell membrane voltage $\left(V_{m}\right.$; middle panel) as the firing field is traversed (top panel), but no change in the amplitude of theta band membrane potential oscillations $\left(M O_{\vartheta}\right)$ across the firing field. However, the CAN model does not account for the theta modulation or phase precession of grid cell firing (data not shown). Panels B-C adapted from Schmidt-Hieber and Häusser, 2013.

The Burak and Fiete (2009) multiple bump CAN model shown in Figure 5A is particularly efficient as it can be implemented as a convolutional neural network of rate-based or simple spiking neurons where, instead of computing the input to each neuron separately, network activity is convolved with the synaptic weight matrix in a single step. A more biophysically realistic single bump implementation using separate layers of excitatory and inhibitory integrate-and-fire neurons has also been shown to produce theta-nested gamma oscillations in model grid cells (Pastoll et al., 2013). CANs consisting of detailed compartmental neurons have not yet been implemented, as simulating several minutes of grid cell firing in a network of $>1000$ model neurons is computationally prohibitive at this time. A simplified approach can be taken, however, where synaptic input rates are first derived from one of the rate-based model neurons and then fed into a single compartmental model neuron in a separate simulation (Schmidt-Hieber and Häusser, 2013; Figure 5B). For example, 
probabilities of firing for excitatory and inhibitory inputs can be derived from one of the rate-based model neurons (according to $P_{\text {exc }}(t, t+\Delta t)=f\left(B_{i}\right) \Delta t$ and $P_{\text {inh }}(t, t+\Delta t)=f\left(-R_{i}\right) \Delta t$, respectively) and used to generate Poisson input spike trains driving excitatory and inhibitory synaptic conductances in a compartmental model, similar to the approach described in Equation 3.

\section{Critique of the Continuous Attractor Network Model}

The CAN model can readily account for the modular organisation of grid cells (Barry et al., 2007; Stensola et al., 2012), and strong functional interactions between grid cells from within the same module in both 1D and 2D environments (Yoon et al., 2013; 2016). For example, the coherent shift in firing field orientation (Hafting et al., 2005; Fyhn et al., 2007) and rescaling (Barry et al., 2007, 2012a; Stensola et al., 2012) of simultaneously recorded grid cells from the same module in 2D suggest that these are functionally coupled. In addition, instabilities in grid cell activity over time apparently correspond to drifts in a stable grid firing pattern relative to the environment, as opposed to corruption of the grid firing pattern itself, consistent with a CAN model (Hardcastle et al., 2015; Chen et al., 2016; Perez-Escobar et al., 2016).

The CAN model predicts the observed ramp depolarisation of grid cells in their firing field (Figure 5C; Domnisoru et al., 2013; Schmidt-Hieber and Häusser, 2013; Bush and Burgess, 2014). Moreover, it has been demonstrated that stellate cells in $\mathrm{mEC}$ exhibit extensive recurrent inhibitory circuitry (Dhillon and Jones, 2000; Couey et al., 2013; Fuchs et al., 2016) that is, in principle, sufficient to mediate continuous attractor dynamics (Burak and Fiete, 2009; Pastoll et al., 2013; ShipstonSharman et al., 2016). In addition, inhibitory inputs to stellate cells in mEC appear around the same time point during development as stable, adult-like grid cell firing patterns (Langston et al. 2010; Wills et al., 2010; Couey et al., 2013). Recent theoretical studies have demonstrated how this recurrent synaptic connectivity might be learned in an unsupervised manner (Widlowski and Fiete, 2014), although it is also possible that some other mechanism is responsible for the initial generation of grid firing patterns (McNaughton et al., 2006; Burgess et al., 2007; Kropff and Treves, 2008; Bush and Burgess, 2014; Dordek et al., 2016). Finally, cells with conjunctive grid $x$ head direction responses, which are required to shift the activity bump in traditional CAN models, have been identified in the deeper layers of mEC (McNaughton et al., 2006; Sargolini et al,. 2006; Navratilova et al., 2012). Similarly, the directionally modulated firing patterns exhibited by grid cells when excitatory drive to $\mathrm{mEC}$ is reduced by inactivation of the hippocampus is consistent with later CAN models (Bonnevie et al., 2013).

Implementations of CAN models with inhibitory disynaptic recurrent connectivity predict that silencing inhibitory interneurons in the $\mathrm{mEC}$ should eliminate grid cell firing patterns, and that the same interneurons should exhibit spatially modulated firing patterns, as they are driven by input from grid cells (Pastoll et al., 2013; Bush and Burgess, 2014). To date, it has been demonstrated that the firing patterns of parvalbumin positive interneurons in $\mathrm{mEC}$, which have strong, recurrent connections with grid cells, tend to show low spatial selectivity and gridness scores; and that these interneurons receive input from grid cells with a wide range of spatial phases (Buetfering et al., 2014). This raises the question of whether they can support continuous attractor dynamics in the grid cell population. Nonetheless, several other classes of interneurons in the local circuits of $\mathrm{mEC}$ could be used to support continuous attractor dynamics, and further experiments are required to 
ascertain whether those neurons exhibit spatially modulated firing patterns or are necessary to support grid cell activity. Moreover, recent theoretical studies have demonstrated that adding spatially uncorrelated noise input to inhibitory neurons in a spiking CAN model (Pastoll et al., 2013) reduces spatial selectivity and impairs grid firing patterns in the interneuron population without compromising those in excitatory cells (Solanka et al., 2015).

Despite this wealth of evidence in support of attractor dynamics in grid cell firing patterns, very few CAN models of grid cell firing can account for theta modulation or phase precession. Those that do rely on subthreshold currents both to maintain the position of the activity bump between theta cycles (Pastoll et al., 2013) and to account for the temporal code of grid cell firing (Navratilova et al., 2012). This solution becomes problematic during periods when the animal is stationary, and grid cells are temporarily inactive or represent distant locations (Olafsdottir et al., 2016). As CAN models encode path integration information in the location of the activity bump, some mechanism must reinstantiate that activity bump in the same location within the network when the animal starts to move again and grid cell firing resumes. In familiar environments, theoretical studies have demonstrated that place or boundary cell input can eliminate drift of the attractor bump over time (Fuhs and Touretzky, 2006; Guanella et al., 2007; Pastoll et al., 2013; Hardcastle et al., 2015). In novel environments, however, where the associations between grid, place and boundary cell responses have not been learned, there is no obvious solution to this problem.

It is also important to consider that grid firing patterns and phase precession may be functionally independent phenomena. For example, grid cells in layer III of the rodent $\mathrm{mEC}$ exhibit a triangular array of firing fields without phase precession, spike times instead being phase locked to the trough of the ongoing theta oscillation (Hafting et al., 2008; Climer et al., 2013; Jeewajee et al., 2014). This raises the possibility that models of grid cell firing need not account for phase precession. However, these data also indicate that the firing of layer III grid cells follows that of layer II grid cells within each theta cycle, suggesting that they may inherit grid firing patterns from generative mechanisms in the more superficial layer (Hafting et al., 2008). In addition, there are - to date - no experimental manipulations that can eliminate grid cell phase precession without also eliminating grid firing patterns, indicating that the two phenomena may be co-dependent.

\section{A Hybrid Grid Cell Model}

The simulations and discussion above illustrate weaknesses in both the OI and CAN models primarily, that the OI model fails to account for functional interactions between grid cell firing patterns, or the subthreshold ramp depolarisation of grid cells in their firing fields; and the CAN model fails to account for the phase precession of grid cell firing in the absence of an additional mechanism. In light of this, it is important to note that these two classes of grid cell model are not mutually exclusive - they each account for different properties of grid firing patterns using different mechanisms and can therefore be reconciled within a single 'hybrid' model (Burgess et al., 2007; Zilli, 2012; Schmidt-Hieber and Häusser, 2013; Bush and Burgess, 2014). We now describe such a model, which makes use of continuous attractor dynamics to ensure relative stability among the firing patterns of grid cells from within the same module and produce subthreshold ramp depolarisation within firing fields; while oscillatory interference is used to shift the activity bump, 
generate phase precession, and store path integration information in VCO phases between theta cycles and when the grid cell network is inactive.

In this implementation, grid cells are modelled as leaky integrate and fire neurons with a membrane time constant of $\tau_{m}={ }^{c_{m}} / g_{m}$ (Equation 7). Simulated neurons integrate current input $I(t)$ until the membrane potential $V_{m}$ reaches a threshold $V_{t}$, at which point a spike is fired and the membrane potential is reset to $V_{\text {reset }}$.

$$
\frac{d V_{m}}{d t}=\frac{1}{c_{m}}\left(I(t)-g_{m}\left(V_{m}-V_{l}\right)\right)
$$

Equation 7: The leaky integrate and fire neuron model. The membrane potential $V_{m}$ of a simulated neuron is dependent on the membrane capacitance $c_{m}$, membrane conductance $g_{m}$, applied current $I$ and leak reversal potential $V_{l}$.

Grid cells receive synaptic input with fixed strength $w_{v c o}$ from six populations of inhibitory VCOs that are arranged in ring attractor circuits (Blair et al., 2008; Welday et al., 2011; Bush and Burgess, 2014). VCOs in each ring attractor circuit share a single preferred firing direction $\emptyset_{V C O}$ but differ in their initial phase $\varphi_{V C O}$. To generate hexagonal grid-like firing patterns, the preferred firing directions of each VCO ring attractor circuit differ by multiples of $60^{\circ}$; and to produce evenly spaced grid fields, the initial phase of VCOs within each ring attractor circuit are uniformly distributed among $N_{\text {offset }}$ values. To produce more realistic membrane dynamics in the grid cell population by increasing the number of inputs, $N_{\text {vcopy }}$ copies of each VCO input (i.e. combination of preferred firing direction and phase) are used.

The burst firing frequency of VCO inputs $f_{V C O}$ increases linearly above a constant baseline oscillation frequency of $f_{\text {base }}=8 \mathrm{~Hz}$ according to movement speed in the preferred direction (as described by Equation 1). Each VCO input produces an inhomogeneous, inhibitory Poissonian spike train only when movement speed in the preferred direction $\mathbf{v}_{\mathrm{vco}}$ is positive, with the probability $p(n, t)$ of firing $n$ spikes in time step $t$ described by Equation 8.

$$
\begin{gathered}
p(n, t)=\frac{\lambda_{V C O}^{n}(t) e^{-\lambda_{V C O}(t)}}{n !} H\left[\mathbf{v}_{V C O}\right] \\
\lambda_{V C O}(t)=\overline{r_{V C O}}\left(\cos \left(2 \pi f_{V C O}(t) t+\varphi_{V C O}\right)+1\right) \Delta t
\end{gathered}
$$

Equation 8: Simulated VCO input spike train in the hybrid model of grid cell firing. The probability of a VCO input firing $n$ spikes in time step $t$ is dictated by the rate function $\lambda_{v c o}$, where $H[x]=0$ for $x<0$ and $H[x]=1$ for $x \geq 0$. The rate function is, in turn, dictated by the mean VCO firing rate $\overline{r_{V C O}}$, burst firing frequency $f_{V C O}$ (see Equation 1), spatial phase offset of that VCO input $\varphi_{V C O}$ and length of the time step $\Delta t$.

Each grid cell in the hybrid network model is recurrently connected to a population of inhibitory interneurons that are also modelled as integrate and fire neurons according to Equation 7. All $N_{\text {Gcopy }}$ grid cells that share a spatial phase send excitatory synapses with strength $w_{G C}$ to a unique subpopulation of $N_{\text {Icopy }}$ interneurons, which subsequently exhibit grid firing patterns with the same 
spatial phase. This interneuron sub-population sends reciprocal projections with strength $w_{I N H}$ to the entire grid cell population with synaptic weights that are a cosine tuned function of their difference in spatial phase to create a 'twisted torus' topology (Figure 4C). Finally, to elicit firing, grid cells receive a tonic excitatory current $I_{\text {exc }}(t)$ that is drawn randomly from a Gaussian distribution with mean $\overline{I_{\text {exc }}}$ and standard deviation $\sigma_{\text {exc }}$ at each time step.

Simulations of the hybrid model demonstrate that it can produce periodic, grid-like firing patterns in one or two dimensional environments (Figure 6). In addition, theta phase precession of firing is observed as each grid field is traversed (Figure 6Bv). Moreover, the combination of rhythmic VCO input and recurrent inhibition can account for the experimentally observed pattern of subthreshold membrane potential dynamics (Domnisoru et al., 2013; Schmidt-Hieber and Häusser, 2013). First, recurrent inhibition hyperpolarises grid cells outside of their firing fields, generating a slow, ramped depolarisation on entry to the firing field (Figure 6Biii). Second, recurrent inhibition is theta modulated - as it is driven by active grid cells with theta modulated firing - and so there is no significant difference in membrane potential theta amplitude in and out of the grid firing field, where subthreshold theta oscillations are driven by VCO inputs and recurrent inhibition, respectively (Figure 6Biv; see Bush and Burgess, 2014 for more details).

\section{Critique of the Hybrid Model}

The hybrid model can account for a wide range of experimental data, including both the rate and temporal firing pattern of grid cells, the relative stability of grid cell firing patterns from the same module, and the subthreshold ramp depolarisation of grid cells inside the firing field. However, the hybrid model also exhibits some weaknesses. Firstly, it offers no explanation for the function of conjunctive cells in the deeper layers of $\mathrm{mEC}$, or for the directional modulation of grid cell firing patterns when excitatory drive from the hippocampus is reduced, as the population activity bump is shifted by input from VCOs. It is possible that redundant mechanisms exist for path integration, and both conjunctive cells or grid cells with directionally modulated input and VCOs are capable of updating grid cell firing during movement. Intriguingly, the majority of conjunctive cells do not show phase precession, suggesting that their firing patterns may be accounted for by a different mechanism (Climer et al., 2013). In its current form, then, the hybrid model predicts that silencing conjunctive cells should have no effect on grid firing patterns, but that silencing VCO inputs should prevent the grid firing pattern from being updated during movement. 
A

(i)

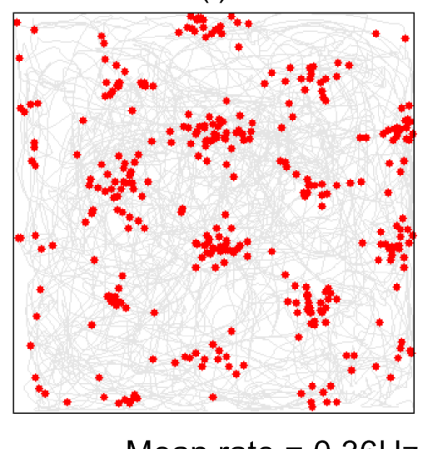

Mean rate $=0.36 \mathrm{~Hz}$ (ii)

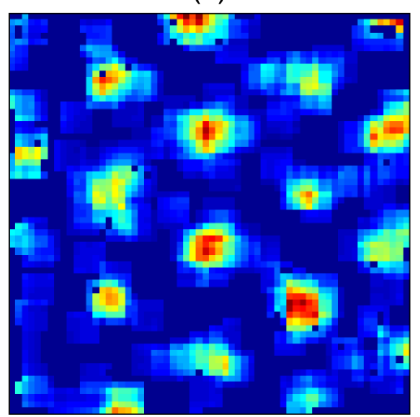

Peak rate $=2.33 \mathrm{~Hz}$ (iii)

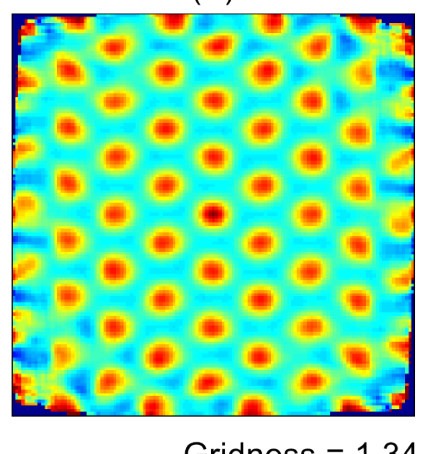

Gridness $=1.34$

B

(i)

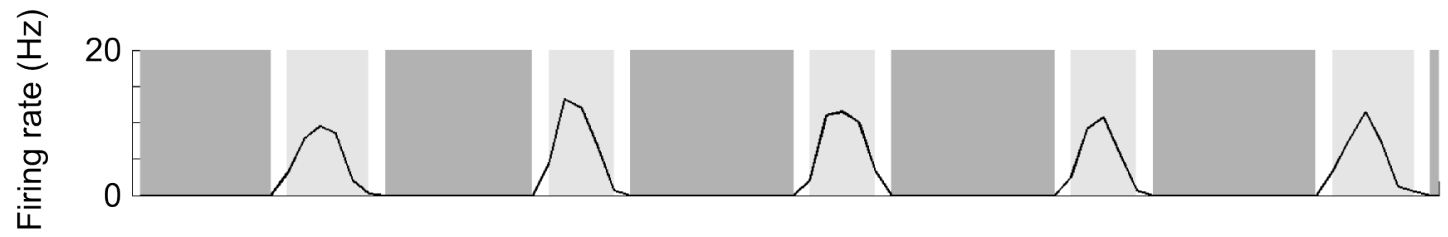

(ii)

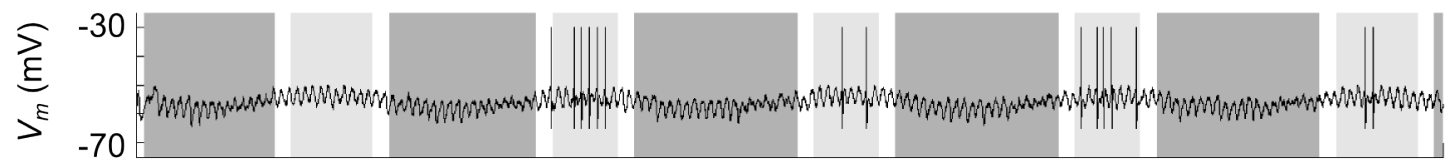

(iii)

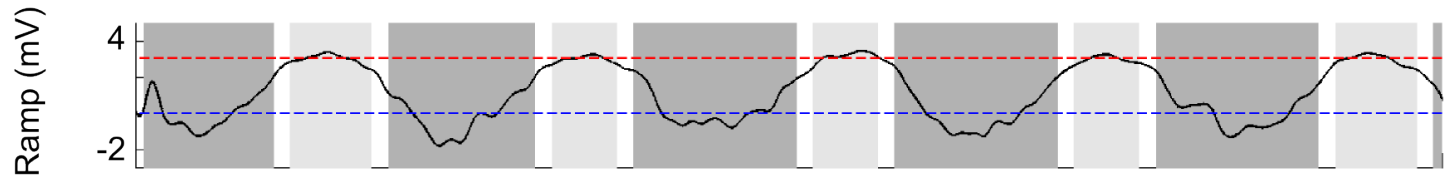

(iv)

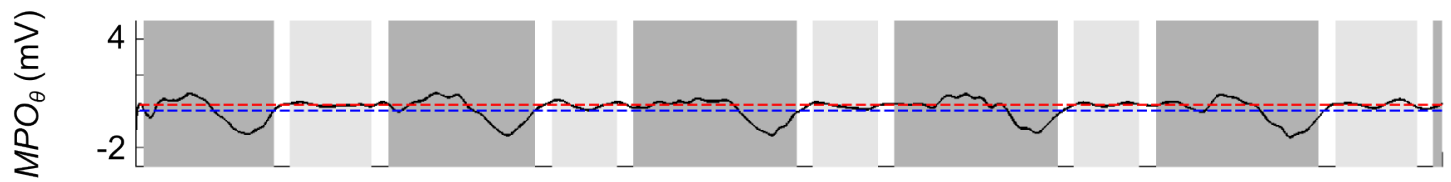

(v)

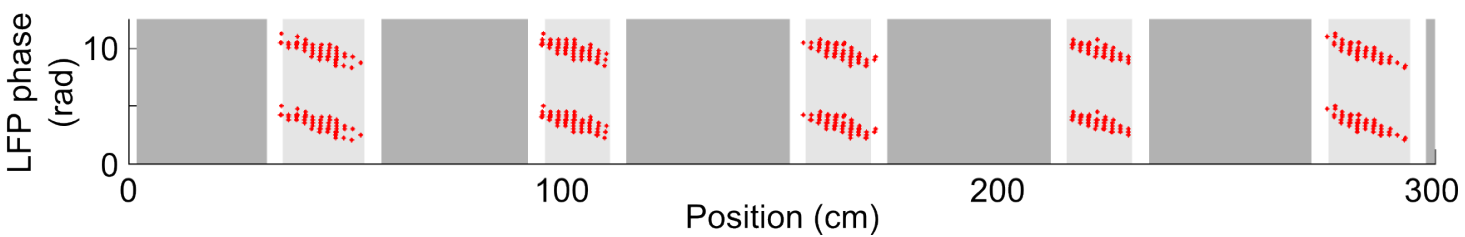

Figure 6: A hybrid oscillatory interference (OI) and continuous attractor network (CAN) model (adapted from Bush and Burgess, 2014). (A) Simulations of the hybrid OI/CAN model in a $1 \mathrm{~m}^{2} 2 \mathrm{D}$ arena. VCO inputs determine the location of the activity bump and integrate movement over time, thereby shifting its location according to self-motion. Synaptic interactions between grid cells couple their firing patterns, providing relative stability. (i) Path taken by the animal (grey) and the location of spikes fired by a typical grid cell (red); (ii) smoothed firing rate map; and (iii) smoothed spatial auto-correlation. (B) Simulations of the hybrid OI/CAN model on a 1D track. (i) Mean grid cell firing rate; (ii) membrane potential of a typical grid cell; (iii) mean low frequency $(<3 \mathrm{~Hz})$ 'ramp' amplitude in the membrane potential; (iv) mean $5-11 \mathrm{~Hz}$ theta amplitude in the membrane potential; and ( $v$ ) phase of firing relative to LFP theta. In- (light grey background) and out- of-field (dark grey background) regions are used to compute mean ramp depolarisation and theta band MPO amplitude inside (red dashed line) and outside (blue dashed line) grid firing fields. The hybrid model predicts a ramped depolarisation of the grid cell membrane voltage as the firing field is traversed, and no change in the amplitude of membrane potential oscillations (MPO) across the firing field, in line with experimental data (Domnisoru and Tank, 2013; Schmidt-Hieber and Häusser, 2013). 
Secondly, the hybrid model - like all other CAN models - predicts that interneurons in circuits local to grid cell populations should exhibit spatially modulated firing patterns, and that silencing those interneurons should impair grid firing patterns. As described above, it has been demonstrated that parvalbumin positive inhibitory cells in $\mathrm{MEC}$ - which are strongly, recurrently connected to grid cells - show low spatial selectivity, low gridness scores, and receive input from grid cells with a wide range of spatial phases (Buetfering et al., 2014). This suggests that this class of interneurons may not be able to support continuous attractor dynamics, although several other classes of interneurons exist in $\mathrm{mEC}$ and further experiments are required to ascertain their firing patterns and relationship to grid cell activity (Solanka et al., 2015). In addition, because grid cells in the hybrid model - unlike the majority of previous CAN models - exhibit phase precession, the interneurons that support continuous attractor dynamics should also exhibit phase precession (Bush and Burgess, 2014). Whether interneurons in $\mathrm{mEC}$ exhibit this temporal code has yet to be established.

Finally, the hybrid model presented here also fails to account for sensory inputs to the grid cell network, which are likely to be important for reducing accumulated error during path integration (Fuhs and Touretzky, 2006; Pastoll et al., 2013; Bush and Burgess, 2014; Evans et al., 2016). Such inputs are straightforward to incorporate into the model, however, by allowing the unsupervised learning of excitatory connections from place or boundary cells to grid cells. It is interesting to note that this spatially modulated input might, in familiar environments, be sufficient to produce a subthreshold ramp depolarisation in grid cells (Domnisoru et al., 2013; Schmidt-Hieber and Häusser, 2013), and correlations in spike-timing at very short time lags between grid cells with similar spatial phases (Tocker et al., 2015).

\section{The Future}

Current experimental and theoretical data highlight several outstanding questions that are critical to the development of the next generation of grid cell models. First, both OI and CAN models assume that the primary function of grid cells is path integration, and thus that grid firing patterns should primarily be accounted for by self-motion information. However, experimental evidence in support of this key assumption is currently lacking. Second, the effect of optogenetically silencing inhibitory interneurons in $\mathrm{mEC}$ on grid cell firing patterns should provide evidence either for or against the assumption, made by CAN models, that grid firing patterns are generated by recurrent inhibitory interactions between grid cells. Similarly, it would be of great interest to ascertain whether grid cells in other species, and in the rodent pre- and para- subiculum, exist within microcircuits of a similar structure, or whether it is possible that independent mechanisms for generating grid firing patterns have evolved in disparate cortical and phylogenetic loci. Third, any mechanism that accounts for the existence of grid cell firing patterns must also provide some explanation for the sudden appearance of stable, adult-like grid cell responses in the rodent $\mathrm{mEC}$ during development. Fourth, optogenetic and juxtacellular recording techniques could shed light on the relationship between grid firing patterns in reelin positive stellate (or "ocean") cells and calbindin positive pyramidal (or "island") cells in $\mathrm{mEC}$, and how this relationship constrains the mechanism that generates grid firing patterns in each of these cell types across development.

Finally, most network-level models of grid cell firing assume that the individual rate-based or integrate-and-fire model neurons have uniform integrative properties and perform simple linear 
transformations of synaptic inputs into action potential output (Equation 4). However, grid cell candidate neurons in $\mathrm{mEC}$ express a rich repertoire of active nonlinear conductances, some of which are tuned to their functional grid cell properties (reviewed in Pastoll et al., 2012; Schmidt-Hieber and Häusser, 2014; Schmidt-Hieber and Nolan, 2017). Moreover, the functional distribution of synaptic inputs on the dendritic tree of grid cells may strongly affect how signals are integrated and transformed into action potential output. Imaging and electrophysiological studies suggest an important function for active dendritic conductances in the generation of place fields in CA1 (Lee et al., 2012; Bittner et al., 2015; Sheffield et al., 2015). Similarly, active dendrites may improve the robustness and precision of the rate and temporal codes of grid cells, suggesting an important role for nonlinear integration in the computation performed by grid cells (Schmidt-Hieber et al., 2017).

It is important to note that current experimental data also presents several challenges to all current models of grid cell firing patterns. First, distortions of the grid pattern close to environmental boundaries (Krupic et al., 2015; Stensola et al., 2015) present an issue to any model that accounts for grid cell firing patterns purely in terms of self-motion inputs, as it implies that an animal's perception of its own motion is perturbed by proximity to environmental boundaries. This issue might be solved by appealing to environmental sensory inputs to grid cells, which are likely to come from boundary cells (Evans et al., 2016; Hardcastle et al., 2015), but the question of why such inputs would actively distort the grid firing pattern, rather than simply reducing accumulated error, remain. It is possible that the observed grid field distortion offers a functional advantage, but if so, this has yet to be identified. Second, the stable differences in firing rate between different grid fields of a single cell are not accounted for by any of the models described above, although these differences may be important to encode contextual information and contribute to place cell remapping (Rolls et al., 2006; Andrzejak and Bicanski, 2007; but see Fyhn et al., 2007). Finally, and most importantly, both OI and CAN models assume that self-motion input is readily available to the grid cell network, and used to update grid firing patterns according to self-motion information. However, the overwhelming majority of single cell responses in and around $\mathrm{mEC}$ encode head direction, not movement direction, even when those two signals differ significantly (Raudies et al., 2015). The importance of movement direction information is reinforced by the demonstration that phase precession follows body movement - rather than head direction - when rats travel backwards through place fields on a linear track (Cei et al., 2014). The origin of the movement direction signal that is clearly required to update grid cell firing patterns in this case, and during normal locomotion, has yet to be identified.

\section{$\underline{\text { Acknowledgements }}$}

The authors would like to thank Andrej Bicanski, Neil Burgess, Talfan Evans, Robin Hayman, Matt Nolan and Freyja Ólafsdóttir for helpful comments and discussion during the preparation of this chapter. 


\section{Resources}

A list of freely available code for various grid cell simulations is given below.

Burak and Fiete (2009) CAN simulations:

http://clm.utexas.edu/fietelab/code.htm

Compilation of various grid cell model implementations by Eric Zilli (Zilli, 2012):

https://senselab.med.yale.edu/modeldb/showModel.cshtml?model=144006

Pastoll et al. (2013) CAN simulations:

https://senselab.med.yale.edu/ModelDB/ShowModel.cshtml?model=150031

Schmidt-Hieber and Häusser (2013) compartmental model:

https://senselab.med.yale.edu/modeldb/showModel.cshtml?model=150239

Solanka et al. (2015) CAN simulations:

https://github.com/MattNolanLab/ei-attractor

Matlab code for hybrid model simulations presented in this chapter:

https://senselab. med.yale.edu/ModelDB/ShowModel.cshtml?model=218085 


\section{$\underline{\text { References }}$}

Alonso A, Llinas RR (1989) Subthreshold $\mathrm{Na}^{+}$-dependent theta-like rhythmicity in stellate cells of entorhinal cortex layer II. Nature 342: 175-177

Andrzejak RG, Bicanski A (2007) Forming place cells through feedforward input from grid cells - a computational model. Society for Neuroscience Abstract 753.1

Barry C, Lever C, Hayman R, Hartley T, Burton S, O'Keefe J, Jeffery K, Burgess N (2006) The boundary vector cell model of place cell firing and spatial memory. Reviews in the Neurosciences 17: 71-97

Barry C, Hayman R, Burgess N, Jeffery K (2007) Experience-dependent rescaling of entorhinal grids. Nature Neuroscience 10: 682-684

Barry C, Ginsberg LL, O'Keefe J, Burgess N (2012a) Grid cell firing patterns signal environmental novelty by expansion. PNAS 109: $17687-17692$

Barry C, Bush D, O’Keefe J, Burgess N (2012) Models of Grid Cells and Theta Oscillations. Nature 488: E1

Bittner KC, Grienberger C, Vaidya SP, Milstein AD, Macklin JJ, Suh J, Tonegawa S, Magee JC (2015) Conjunctive input processing drives feature selectivity in hippocampal CA1 neurons. Nature Neuroscience 18: 1133-1142

Blair HT, Gupta K, Zhang K (2008) Conversion of a phase- to a rate-coded position signal by a three stage model of theta cells, place cells, and grid cells. Hippocampus 18: 1239-55

Blair HT, Wu D, Cong J (2014). Synchronization coding by ring attractors: A theoretical framework for oscillatory neurocomputing. Phil Trans R Soc Lond B Biol Sci 369: 20120526

Boccara CN, Sargolini F, Thoresen VH, Solstad T, Witter MP, Moser El, Moser MB (2010) Grid cells in pre- and parasubiculum. Nature Neuroscience 13: 987 - 994

Bonnevie T, Dunn B, Fyhn M, Hafting T, Derdikmann D, Kubie JL, Roudi Y, Moser El, Moser M-B (2013) Grid cells require excitatory drive from the hippocampus. Nature Neuroscience 16: 309-317

Brandon MP, Bogaard AR, Libby CP, Connerney MA, Gupta K, Hasselmo ME (2011) Reduction of theta rhythm dissociates grid cell spatial periodicity from directional tuning. Science 332: 595-599

Buetfering C, Allen K, Monyer H (2014) Parvalbumin interneurons provide grid cell-driven recurrent inhibition in the medial entorhinal cortex. Nature Neuroscience 17: 710-718

Burak Y, Fiete IR (2009) Accurate path integration in continuous attractor network models of grid cells. PLoS Computational Biology 5: e1000291

Burgess N, Barry C, Jeffery KJ, O'Keefe J (2005) A grid and place cell model of path integration utilizing phase precession versus theta. Computational Cognitive Neuroscience Conference Poster; Washington, DC: http://f1000.com/posters/browse/summary/225 
Burgess N, Barry C, O'Keefe J (2007) An oscillatory interference model of grid cell firing. Hippocampus 17: $801-812$

Burgess N (2008) Grid cells and theta as oscillatory interference: Theory and predictions. Hippocampus 18: 1157 - 1174

Burgess CP, Burgess N (2014) Controlling phase noise in oscillatory interference models of grid cell firing. Journal of Neuroscience 34: 6224-6232

Bush D, Burgess N (2014). A hybrid oscillatory interference/continuous attractor network model of grid cell firing. Journal of Neuroscience 34: 5065-5079

Bush D, Barry C, Manson D, Burgess N (2015) Using Grid Cells for Navigation. Neuron 87: 507-520

Cei A, Girardeau G, Drieu C, El Kanbi K, Zugaro M (2014) Reversed theta sequences of hippocampal cell assemblies during backward travel. Nature Neuroscience 17: 719-724

Chen G, Manson D, Cacucci F, Wills TJ (2016) Absence of Visual Input Results in the Disruption of Grid Cell Firing in the Mouse. Current Biology (in press)

Climer JR, Newman EL, Hasselmo ME (2013) Phase coding by grid cells in unconstrained environments: two-dimensional phase precession. European Journal of Neuroscience 38: 2526-2541

Conklin J, Eliasmith C (2005) An attractor network model of path integration in the rat. Journal of Computational Neuroscience 18: 183-203

Couey JJ, Witoelar A, Zhang S-J, Zheng K, Ye J, Dunn B, Czajkowski R, Moser M-B, Moser El, Roudi Y, Witter MP (2013) Recurrent inhibitory circuitry as a mechanism for grid formation. Nature Neuroscience 16: 318-324

Derdikman D, Whitlock JR, Tsao A, Fyhn M, Hafting T, Moser M-B, Moser El (2009) Fragmentation of grid cell maps in multicompartment environment. Nature Neuroscience 12: 1325-1332

Dhillon A, Jones R (2000) Laminar differences in recurrent excitatory transmission in the rat entorhinal cortex in vitro. Neuroscience 99: 413-422.

Doeller CF, Barry C, Burgess, N (2010) Evidence for grid cells in a human memory network. Nature 463 657- 661

Dordek Y, Soudry D, Meir R, Derdikman D (2016) Extracting grid cell characteristics from place cell inputs using non-negative principal component analysis. eLife 5: e10094

Domnisoru C, Kinkhabwala AA, Tank DW (2013) Membrane potential dynamics of grid cells. Nature 495: 199-204

Eliav T, Geva-Sagiv M, Finkelstein A, Yartsev M, Rubin A, Las L, Ulanovksy N (2015) Synchronicity without rhythmicity in the hippocampal formation of behaving bats. Society for Neuroscience Abstract 632.01 
Ekstrom AD, Caplan JB, Shattuck K, Fried I, Kahana MJ (2005) Human hippocampal theta activity during virtual navigation. Hippocampus 15: 881-889

Evans T, Bicanski A, Bush D, Burgess N, (2016) How environment and self-motion combine in neural representations of space. Journal of Physiology (in press)

Fiete IR, Burak $Y$, Brookings $T$ (2008) What grid cells convey about rat location. Journal of Neuroscience 28: 6858-6871

Fuchs EC, Neitz A, Pinna R, Melzer S, Caputi A, Monyer H (2016) Local and Distant Input Controlling Excitation in Layer II of the Medial Entorhinal Cortex. Neuron 89: 194-208

Fuhs MC, Touretzky DS (2006) A spin glass model of path integration in rat medial entorhinal cortex. Journal of Neuroscience 26: $4266-4276$

Fyhn M, Hafting T, Treves A, Moser M-B, Moser El (2007) Hippocampal remapping and grid realignment in entorhinal cortex. Nature 446: 190-194

Fyhn M, Hafting T, Witter MP, Moser El, Moser MB (2008) Grid cells in mice. Hippocampus 18: 1230 1238

Garden DLF, Dodson PD, O'Donnell C, White MD, Nolan MF (2008) Tuning of Synaptic Integration in the Medial Entorhinal Cortex to the Organization of Grid Cell Firing Fields. Neuron 60: 875-889

Gatome CW, Slomianka L, Lipp HP, Amrein I (2010) Number estimates of neuronal phenotypes in layer II of the medial entorhinal cortex of rat and mouse. Neuroscience 170: 156-65

Giocomo LM, Zilli EA, Fransén E, Hasselmo ME (2007) Temporal frequency of subthreshold oscillations scales with entorhinal grid cell field spacing. Science 315: 1719-1722

Giocomo LM, Moser M-B, Moser El (2011) Computational Models of Grid Cells. Neuron 71: 589-603

Guanella A, Kiper D, Verschure P (2007) A model of grid cells based on a twisted torus topology. International Journal of Neural Systems 17: 231-240

Hafting T, Fyhn M, Molden S, Moser MB, Moser El (2005) Microstructure of a spatial map in the entorhinal cortex. Nature 436: 801 - 806

Hafting T, Fyhn M, Bonnevie T, Moser MB, Moser El (2008) Hippocampus-independent phase precession in entorhinal grid cells. Nature 453: 1248-52

Hardcastle K, Ganguli S, Giocomo LM (2015). Environmental boundaries as an error correction mechanism for grid cells. Neuron 86: 1-13

Hasselmo ME (2008) Grid cell mechanisms and function: Contributions of entorhinal persistent spiking and phase resetting. Hippocampus 18: $1116-1126$

Heys JG, Rangarajan KV, Dombeck DA (2014) The functional micro-organization of grid cells revealed by cellular-resolution imaging. Neuron 84: 1079-1090 
Horiuchi TK, Moss CF (2015) Grid cells in 3-D: Reconciling data and models. Hippocampus 25: 14891500

Jacobs J, Weidemann CT, Miller JF, Solway A, Burke JF, Wei X, Suthana N, Sperling MR, Sharan AD, Fried I, Kahana MJ (2013) Direct recordings of grid-like neuronal activity in human spatial navigation. Nature Neuroscience 16: 1188-1190

Jeewajee A, Barry C, O'Keefe J, Burgess N (2008) Grid cells and theta as oscillatory interference: electrophysiological data from freely-moving rats. Hippocampus 18: $1175-1185$

Jeewajee A, Barry C, Douchamps V, Manson D, Lever C, Burgess N (2014) Theta phase precession of grid and place cell firing in open environments. Philosophical Transactions of the Royal Society $B$ 369: 20120532

Killian NJ, Jutras MJ, Buffalo EA (2012) A map of visual space in the primate entorhinal cortex. Nature 491: 761-764

Kitamura T1, Pignatelli M, Suh J, Kohara K, Yoshiki A, Abe K, Tonegawa S (2014) Island cells control temporal association memory. Science 343: 896-901

Koenig J, Linder AN, Leutgeb JK, Leutgeb S (2011) The spatial periodicity of grid cells is not sustained during reduced theta oscillations. Science 332: 592-595

Kropff E, Treves A (2008) The emergence of grid cells: intelligent design or just adaptation? Hippocampus 18: 1256-1269

Krupic J, Bauza M, Burton S, Barry C, O'Keefe J (2015) Grid cell symmetry is shaped by environmental geometry. Nature 518: 232-235

Langston RF, Ainge JA, Couey JJ, Canto CB, Bjerknes TL, Witter MP, Moser EI, Moser MB (2010) Development of the spatial representation system in the rat. Science 328: 1576-1580

Lee D, Lin BJ, Lee AK (2012) Hippocampal place fields emerge upon single-cell manipulation of excitability during behavior. Science $337: 849-853$

Lengyel M, Szatmáry Z, Érdi P (2003) Dynamically detuned oscillations account for the coupled rate and temporal code of place cell firing. Hippocampus 13: $700-714$

Lever C, Burton S, Jeewajee A, O'Keefe J, Burgess N (2009) Boundary vector cells in the subiculum of the hippocampal formation. Journal of Neuroscience 29: 9771-9777

McFarland WL, Teitelbaum H, Hedges EK (1975) Relationship between hippocampal theta activity and running speed in the rat. Journal of Comparative and Physiological Psychology 88: 324-328

McNaughton BL, Battaglia FP, Jensen O, Moser El, Moser MB (2006) Path integration and the neural basis of the cognitive map. Nature Reviews Neuroscience 7: $663-678$

Mhatre H, Gorchetchnikov A, Grossberg S (2012) Grid cell hexagonal patterns formed by fast selforganized learning within entorhinal cortex. Hippocampus 22: 320-334 
Naumann RK, Ray S, Prokop S, Las L, Heppner FL, Brecht M (2015) Conserved size and periodicity of pyramidal patches in layer 2 of medial/caudal entorhinal cortex. Journal of Comparative Neurology 524: 783-806

Navratilova Z, Giocomo LM, Fellous JM, Hasselmo ME, McNaughton BL (2012) Phase precession and variable spatial scaling in a periodic attractor map model of medial entorhinal grid cells with realistic after-spike dynamics. Hippocampus 22: 772-789

O'Keefe J, Nadel L (1978) The Hippocampus as a Cognitive Map. Oxford University Press, Oxford, UK

O'Keefe J, Recce ML (1993) Phase relationship between hippocampal place units and the EEG theta rhythm. Hippocampus 3: 317-30

Ólafsdóttir HF, Carpenter F, Barry C (2016) Coordinated grid and place cell replay during rest. Nature Neuroscience 19: 792-794

Orchard J (2015) Oscillator-Interference Models of Path Integration Do Not Require Theta Oscillations. Neural Computation 27: 548-560

Pastoll H, Ramsden H Nolan MF (2012) Intrinsic electrophysiological properties of entorhinal cortex stellate cells and their contribution to grid firing fields. Frontiers in Neural Circuits 6: 1-21

Pastoll H, Solanka L, van Rossum MCW, Nolan MF (2013) Feedback inhibition enables theta-nested gamma oscillations and grid firing fields. Neuron 77: 141-154

Pérez-Escobar JA, Kornienko O, Latuske P, Kohler L, Allen K (2016) Visual landmarks sharpen grid cell metric and confer context specificity to neurons of the medial entorhinal cortex. eLife (in press)

Raudies F, Brandon MP, Chapman GW, Hasselmo ME (2015) Head direction is coded more strongly than movement direction in a population of entorhinal neurons. Brain Research 1621: 355-367

Ray S, Naumann R, Burgalossi A, Tang Q, Schmidt H, Brecht M (2014) Grid-layout and thetamodulation of layer 2 pyramidal neurons in medial entorhinal cortex. Science 343: 891-896

Reifenstein ET, Kempter R, Schreiber S, Stemmler MB, Herz AV (2012) Grid cells in rat entorhinal cortex encode physical space with independent firing fields and phase precession the single-trial level. PNAS 109: 6301-6306

Reifenstein E, Stemmler M, Herz AVM, Kempter R, Schreiber S (2014) Movement Dependence and Layer Specificity of Entorhinal Phase Precession in Two-Dimensional Environments. PLoS One 9: e100638

Remme MW, Lengyel M, Gutkin BS (2010) Democracy-independence trade-off in oscillating dendrites and its implications for grid cells. Neuron 66: 429-437

Rivas J, Gaztelu JM, García-Austt E (1996) Changes in hippocampal cell discharge patterns and theta rhythm spectral properties as a function of walking velocity in the guinea pig. Experimental Brain Research 108: 113-118 
Rolls ET, Stringer SM, Elliot T (2006) Entorhinal cortex grid cells can map to hippocampal place cells by competitive learning. Network: Computation in Neural Systems 447: 447-465

Samsonovich A, McNaughton BL (1997) Path Integration and Cognitive Mapping in a Continuous Attractor Neural Network Model. Journal of Neuroscience 17: 5900 - 5920

Sargolini F, Fyhn M, Hafting T, McNaughton BL, Witter MP, Moser MB, Moser El (2006) Conjunctive representation of position, direction, and velocity in entorhinal cortex. Science 312: 758-62

Savelli F, Yoganarasimha D, Knierim JJ (2008) Influence of boundary removal on the spatial representations of the medial entorhinal cortex. Hippocampus 18: 1270-1282

Schmidt-Hieber C, Häusser M (2013) Cellular mechanisms of spatial navigation in the medial entorhinal cortex. Nature Neuroscience 16: 325-31

Schmidt-Hieber C, Häusser M (2014) How to build a grid cell. Phil Trans Roy Soc B 369: 20120520

Schmidt-Hieber C, Toleikyte G, Aitchison L, Roth A, Clark BA, Branco T, Häusser M (2017) Active dendritic integration as a mechanism for robust and precise grid cell firing. Nature Neuroscience 20:1114-1121

Schmidt-Hieber C, Nolan MF (2017) Synaptic integrative mechanisms for spatial cognition. Nature Neuroscience 26:1483-1492

Sheffield ME, Dombeck DA (2015) Calcium transient prevalence across the dendritic arbour predicts place field properties. Nature 517: 200-204

Shipston-Sharman O, Solanka L, Nolan MF (2016) Continuous attractor network models of grid cell firing based on excitatory-inhibitory interactions. J Physiol doi:10.1113/JP270630

Solanka L, van Rossum MCW, Nolan MF (2015) Noise promotes independent control of gamma oscillations and grid firing within a recurrent attractor network. eLife 4: e06444

Solstad T, Boccara CN, Kropff E, Moser MB, Moser El (2008) Representation of geometric borders in the entorhinal cortex. Science 322: 1865-1868

Stemmler M, Mathis A, Herz AVM (2015) Connecting multiple spatial scales to decode the population activity of grid cells. Science Advances 1: e1500816

Stensola H, Stensola T, Solstad T, Frøland K, Moser MB, Moser El (2012) The entorhinal grid map is discretized. Nature 492: 72-78

Stensola T, Stensola H, Moser M-B, Moser El (2015) Shearing-induced asymmetry in entorhinal grid cells. Nature 518: 207-212

Sun C, Kitamura T, Yamamoto J, Martin J, Pignatelli M, Kitch LJ, Schnitzer MJ, Tonegawa S (2015) Distinct speed dependence of entorhinal island and ocean cells, including respective grid cells. PNAS 112: 9466-9471 
Tocker G, Barak O, Derdikman D (2015) Grid cells correlation structure suggests organized feedforward projections into superficial layers of the medial entorhinal cortex. Hippocampus 25: 1599-1613

Vanderwolf $\mathrm{CH}$ (1969) Hippocampal electrical activity and voluntary movement in the rat. EEG Clinical Neurophysiology 26: $407-418$

Watrous AJ, Lee DJ, Izadi A, Gurkoff GG, Shahlaie K, Ekstrom AD (2013) A comparative study of human and rat hippocampal low-frequency oscillations during spatial navigation. Hippocampus 23: 656-661

Welday AC, Shlifer IG, Bloom ML, Zhang K, Blair HT (2011) Cosine directional tuning of theta cell burst frequencies: evidence for spatial coding by oscillatory interference. Journal of Neuroscience 31: 16157-16176

Welinder PE, Burak Y, Fiete IR (2008) Grid cells: the position code, neural network models of activity, and the problem of learning. Hippocampus 18: 1283-1300

Wells CE, Amos DP, Jeewajee A, Douchamps V, Rodgers RJ, O'Keefe J, Burgess N, Lever C (2013) Novelty and anxiolytic drugs dissociate two components of hippocampal theta in behaving rats. Journal of Neuroscience 33: 8650-8667

Widlowski J, Fiete IR (2014) A Model of Grid Cell Development through Spatial Exploration and Spike Time-Dependent Plasticity. Neuron 83: 481-495

Wills TJ, Cacucci F, Burgess N, O'Keefe J (2010) Development of the hippocampal cognitive map in pre-weanling rats. Science 328: 1573-1576

Winter SS, Mehlman ML, Clark BJ, Taube JS (2015) Passive Transport Disrupts Grid Signals in the Parahippocampal Cortex. Current Biology 25: 2493-2502

Yartsev MM, Witter MP, Ulanovsky N (2011) Grid cells without theta oscillations in the entorhinal cortex of bats. Nature 479: $103-107$

Yoon K, Buice MA, Barry C, Hayman R, Burgess N, Fiete IR (2013) Specific evidence of lowdimensional continuous attractor dynamics in grid cells. Nature Neuroscience 16: 1077-1084

Yoon K, Lewallen S, Kinkhabwala AA, Tank DW, Fiete IR (2016) Grid Cell Responses in 1D Environments Assessed as Slices through a 2D Lattice. Neuron 89: 1086-1099

Zhang K (1996) Representation of spatial orientation by the intrinsic dynamics of the head-direction cell ensemble: a theory. Journal of Neuroscience 16: 2112 - 2126

Zilli EA (2012) Models of grid cell spatial firing published 2005-2011. Frontiers in Neural Circuits 6: 16 